\title{
Geometric realization of locally regular heptagonal toroidal polyhedra.
}

\author{
Ruslan Mizhaev \\ (mimodart@gmail.com)
}

\begin{abstract}
The article presents a geometric realization of toroidal polyhedra whose faces are flat heptagons. As an example, we have analyzed in detail a polyhedron of the 7th genus, consisting of 72 heptagonal faces. This solution allows realization for the number of faces $f \geq 24$ of the genus $g \geq 3$. Polyhedra in this case are closed orientable surfaces with flat faces without self-intersections, consisting of polygons and embedded in a three-dimensional space.
\end{abstract}

\section{Introduction}

At present, problems related to the geometric realization of toroidal polyhedra remain quite relevant. Polyhedra, each face of which is a $p$-gons are of much interest in this area. It should be noted that faces can be convex and non-convex polygons. For cases of hexagonal faces, numerous solutions, described in various articles $[2,4]$, are presented.

Before construction, for convenience, preliminary calculations were performed to determine the characteristics of the desired polyhedra by methods from the field of topology and combinatorics. According to Euler's theorem, the vertices, edges, and faces of a polyhedron of the genus- $g$ are related by the equation:

$$
v-e+f=\chi=2-2 g
$$

where $\chi$ is the Euler characteristic.

For cases where the degrees of all vertices are $\operatorname{deg}(v)=3$, the equality $3 v=2 e$ holds [1]. In this case, we consider the graph embedding in the surface of the genus $-g$. The degrees of the vertices are abbreviated as $-q$.

Since for regular polyhedra of degree of all vertices $q=3$ and the number of vertices of all faces for the cases under consideration are the same $(p=7)$, the number of vertices and edges can be written as $v=\frac{f \cdot p}{3}$, $e=\frac{f \cdot p}{2}$. Substituting these values in equation (1), we obtain the following formulas for calculations

$$
\begin{gathered}
f=12 g-12 \\
g=\frac{f+12}{12}
\end{gathered}
$$

The calculation results for some of the first values are summarized in table 1. 
Table 1

\begin{tabular}{|c|c|c|c|c|}
\hline Genus, $\boldsymbol{g}$ & Faces, $\boldsymbol{f}$ & Vertices, $\boldsymbol{v}$ & Edges, $\boldsymbol{e}$ & Angles, $\boldsymbol{p}$ \\
\hline $\mathbf{2}$ & 12 & 28 & 42 & 7 \\
\hline $\mathbf{3}$ & 24 & 56 & 84 & 7 \\
\hline $\mathbf{4}$ & 36 & 84 & 126 & 7 \\
\hline $\mathbf{5}$ & 48 & 112 & 168 & 7 \\
\hline $\mathbf{6}$ & 60 & 140 & 210 & 7 \\
\hline $\mathbf{7}$ & 72 & 168 & 252 & 7 \\
\hline $\mathbf{8}$ & 84 & 196 & 294 & 7 \\
\hline $\mathbf{9}$ & 96 & 224 & 336 & 7 \\
\hline 10 & 108 & 252 & 378 & 7 \\
\hline 12 & 120 & 280 & 420 & 7 \\
\hline 14 & 132 & 308 & 462 & 7 \\
\hline
\end{tabular}

The polyhedron at $g=2$ was first introduced and described in the article "A locally regular heptagondodecahedron embedded in 3-space" by Jorg M. Wills [3]. Also, a lot of work in this area was done by David I. McCooey, who described new combinations for $g=2$. In addition, he first introduced an realization for $g=3,5,11[5,6]$.

\section{Polyhedra description}

A distinctive feature of the solution presented in the article is the possibility of geometric realization for all cases of this series at $f \geq 24$ and $g \geq 3$. For ease of understanding and representation, polyhedra are divided into condition al segments.

Segments are those sections whose angle $\left(\alpha_{1}\right)$ changes depending on the type of polyhedron and in these examples $\alpha_{1}=360 /(g-1)$ (Fig. 5). Also, polyhedra are symmetrical when rotated by an angle $\alpha_{1}$ around the $\mathrm{OZ}$ axis.

As a detailed example, we will analyze the case $(p, q ; g)(7,3 ; 7)$ in two different constructions, for the other cases, images and brief descriptions are provided, since they are made by analogy. For clarity of general designs, some angles between edges were made small, but they can be seen when considering face layouts on the plane and on general isometric views.

\section{Polyhedron $(7,3 ; 7)-\mathrm{V} 1$.}

This polyhedron has six horizontal 2-fold figure axes and one 6-fold figure axis (Fig. 5), hence the polyhedron has rotational symmetry. The symmetry group for Schoenflies notations - D6.

The angles between the segments $\alpha_{1}=60^{\circ}$ and the center point $O(0: 0: 0)$ are located at the intersection of the figure axes. The polyhedron faces are divided into 6 identical types, 12 of each type (Fig. 4). 


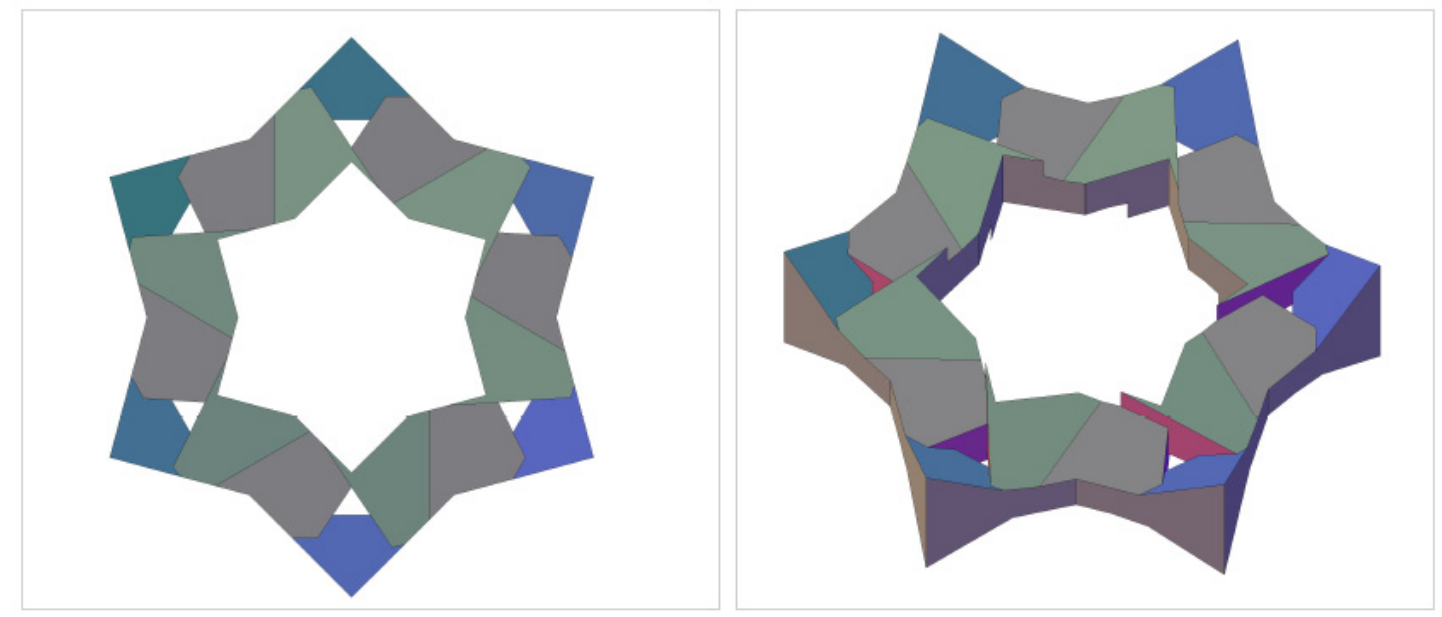

Fig. 1. General views $(7,3 ; 7)-V 1$.
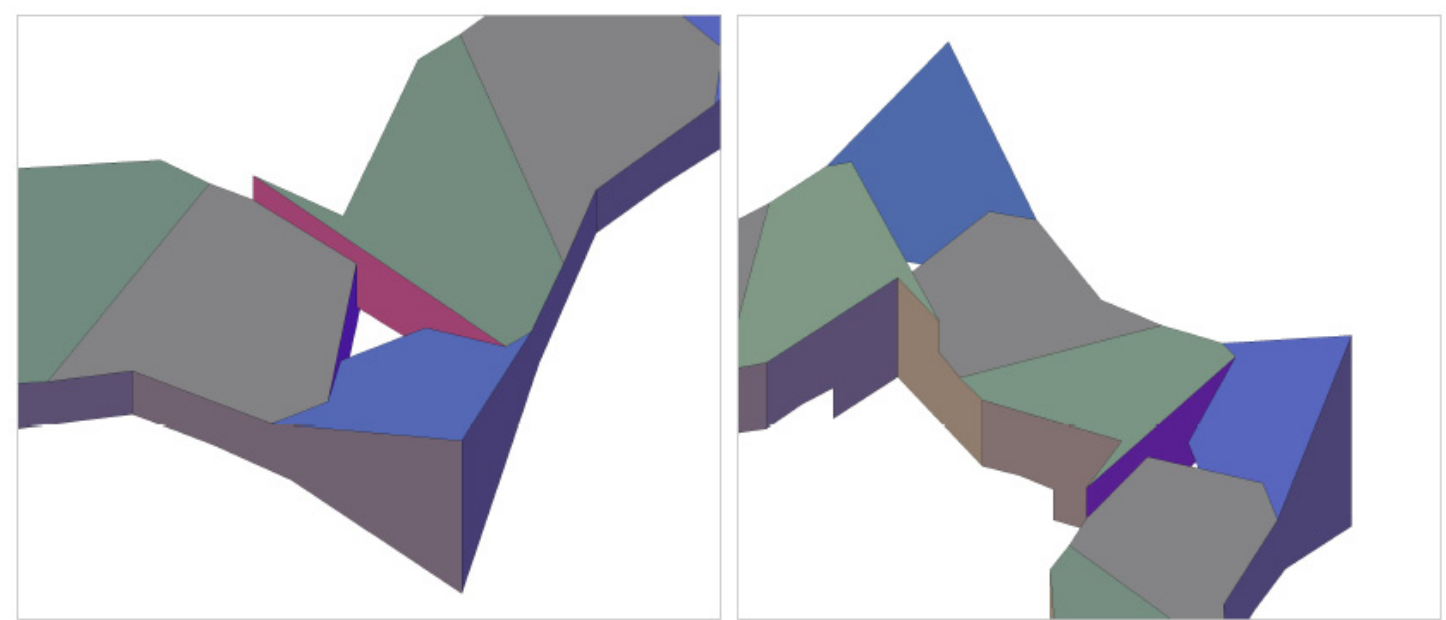

Fig. 2. Sections with an enlarged scale $(7,3 ; 7)-\mathrm{V} 1$.
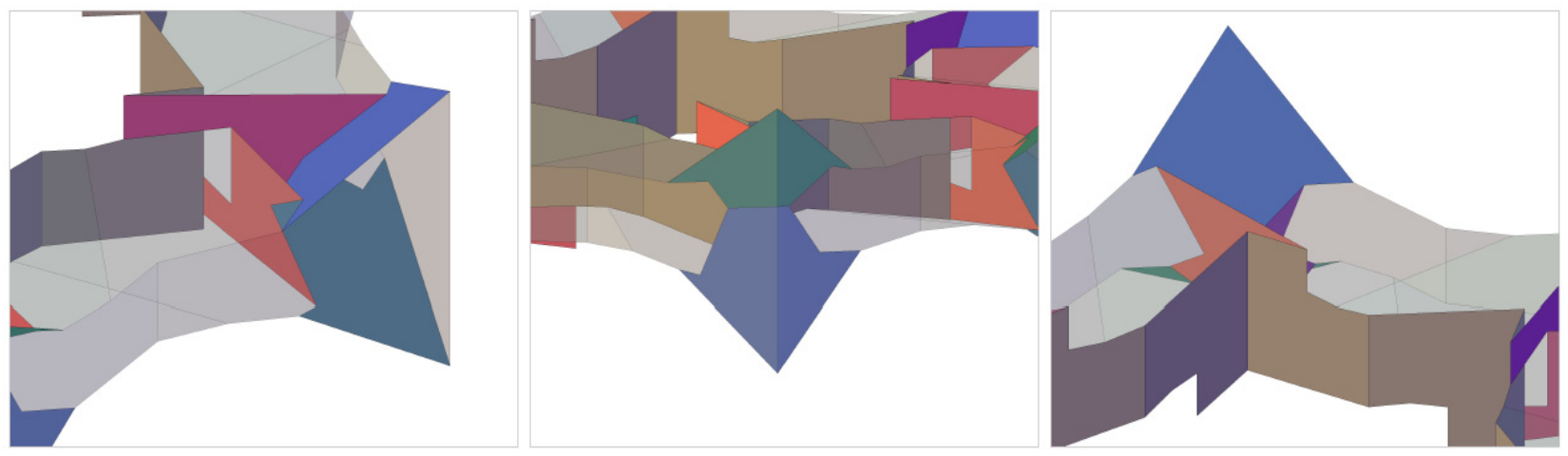

Fig. 3. Sections with enlarged scale and transparent faces $(7,3 ; 7)-\mathrm{V} 1$.

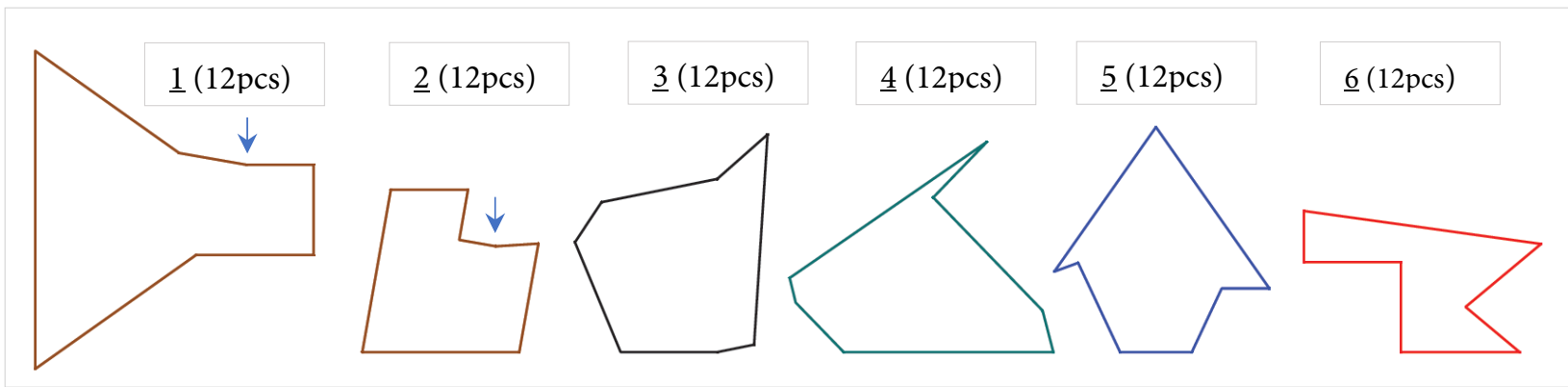

Fig. 4. Layout of faces on the plane $(7,3 ; 7)-\mathrm{V} 1$. 

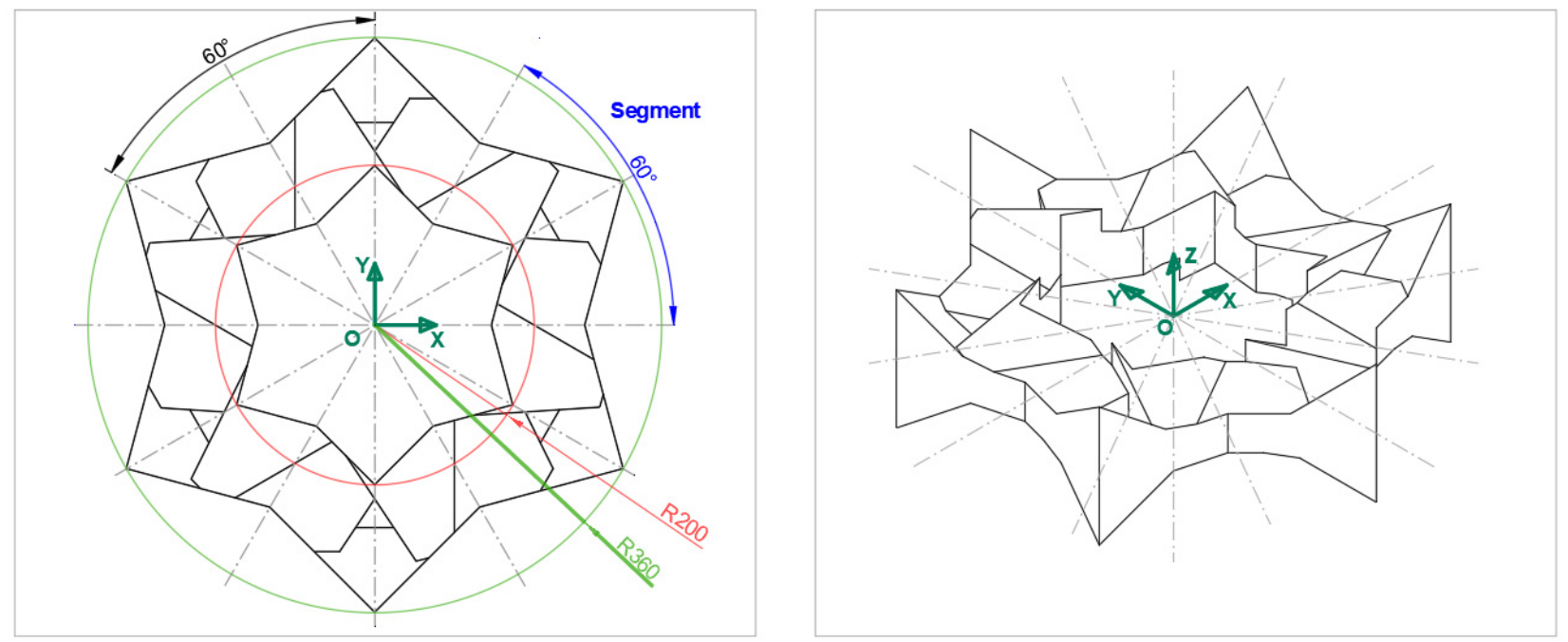

Fig. 5. Dividing the polyhedron into segments $(7,3 ; 7)-\mathrm{V} 1$.

\section{Equations of face planes $(7,3 ; 7)-\mathrm{V} 1$.}

Face planes $\underline{1}$ (Fig. 4 ).

$x+y+360=0$

$x(\sqrt{3}-1)+y(\sqrt{3}+1)+720=0$

$x(\sqrt{3}+1)-y(\sqrt{3}-1)+720=0$

$x(\sqrt{3}+1)+y(\sqrt{3}-1)+720=0$

$x(\sqrt{3}-1)-y(\sqrt{3}+1)+720=0$

$x-y+360=0$

$x+y-360=0$

$x(\sqrt{3}-1)+y(\sqrt{3}+1)-720=0$

$x(\sqrt{3}+1)-y(\sqrt{3}-1)-720=0$

$x(\sqrt{3}+1)+y(\sqrt{3}-1)-720=0$

$x(\sqrt{3}-1)-y(\sqrt{3}+1)-720=0$

$x-y-360=0$

Face planes $\underline{4}$ (Fig. 4 ).

$x+4 z-220=0$

$x-\sqrt{3} y+8 z-440=0$

$x+\sqrt{3} y-8 z+440=0$

$x-4 z+220=0$

$x-\sqrt{3} y-8 z+440=0$

$x+\sqrt{3} y+8 z-440=0$

$x+4 z+220=0$

$x-\sqrt{3} y+8 z+440=0$

$x+\sqrt{3} y-8 z-440=0$

$x-4 z-220=0$

$x-\sqrt{3} y-8 z-440=0$

$x+\sqrt{3} y+8 z+440=0$
Face planes $\underline{2}$ (Fig. 4 ).

$x+y+200=0$

$x(\sqrt{3}-1)+y(\sqrt{3}+1)+400=0$

$x(\sqrt{3}+1)-y(\sqrt{3}-1)+400=0$

$x(\sqrt{3}+1)+y(\sqrt{3}-1)+400=0$

$x(\sqrt{3}-1)-y(\sqrt{3}+1)+400=0$

$x-y+200=0$

$x+y-200=0$

$x(\sqrt{3}-1)+y(\sqrt{3}+1)-400=0$

$x(\sqrt{3}+1)-y(\sqrt{3}-1)-400=0$

$x(\sqrt{3}+1)+y(\sqrt{3}-1)-400=0$

$x(\sqrt{3}-1)-y(\sqrt{3}+1)-400=0$

$x-y-200=0$

Face planes $\underline{5}$ (Fig. 4 ).

$y+z+254=0$

$y-z+254=0$

$y+2 z+\sqrt{3} x+508=0$

$y-2 z+\sqrt{3} x+508=0$

$2 z-y+\sqrt{3} x+508=0$

$\sqrt{3} x-2 z-y+508=0$

$y-z-254=0$

$y+z-254=0$

$y-2 z+\sqrt{3} x-508=0$

$y+2 z+\sqrt{3} x-508=0$

$\sqrt{3} x-2 z-y-508=0$

$2 z-y+\sqrt{3} x-508=0$
Face planes $\underline{3}$ (Fig. 4).

$z-30=0$

$z+30=0$
Face planes $\underline{6}$ (Fig. 4).

$3 x+2 y+436=0$

$3 x-2 y-436=0$

$x(2 \cdot \sqrt{3}+3)-y(3 \cdot \sqrt{3}-2)+872=0$

$x(2 \cdot \sqrt{3}-3)+y(3 \cdot \sqrt{3}+2)+872=0$

$x(2 \cdot \sqrt{3}-3)-y(3 \cdot \sqrt{3}+2)+872=0$

$x(2 \cdot \sqrt{3}+3)+y(3 \cdot \sqrt{3}-2)+872=0$

$3 x+2 y-436=0$

$3 x-2 y+436=0$

$x(2 \cdot \sqrt{3}+3)-y(3 \cdot \sqrt{3}-2)-872=0$

$x(2 \cdot \sqrt{3}-3)+y(3 \cdot \sqrt{3}+2)-872=0$

$x(2 \cdot \sqrt{3}-3)-y(3 \cdot \sqrt{3}+2)-872=0$

$x(2 \cdot \sqrt{3}+3)+y(3 \cdot \sqrt{3}-2)-872=0$ 
Coordinates of polyhedron vertices $(7,3 ; 7)-\mathrm{V} 1$.

The coordinates of the polyhedron vertices are given in the Cartesian coordinate system and divided into 2 columns with the results of symbolic expressions and decimals. For decimals, the last digit is rounded.

Table 2

\begin{tabular}{|c|c|c|c|c|c|c|}
\hline \multirow[t]{2}{*}{ № } & \multicolumn{2}{|c|}{ Coordinates X } & \multicolumn{2}{|c|}{ Coordinates $\mathrm{Y}$} & \multicolumn{2}{|c|}{ Coordinates Z } \\
\hline & $\begin{array}{l}\text { Symbolic } \\
\text { expression }\end{array}$ & Decimals & $\begin{array}{l}\text { Symbolic } \\
\text { expression }\end{array}$ & Decimals & $\begin{array}{l}\text { Symbolic } \\
\text { expression }\end{array}$ & Decimals \\
\hline 1 & $-(146 \cdot \sqrt{ } 3-34)$ & $-218,8794179051$ & $-(34 \cdot \sqrt{3}+146)$ & $-204,8897274573$ & 38 & 38 \\
\hline 2 & $-(130 \cdot \sqrt{ } 3-50)$ & $-175,1666049840$ & $-(50 \cdot \sqrt{3}+130)$ & $-216,602540378$ & 30 & 30 \\
\hline 3 & $-(400-200 \cdot \sqrt{ } 3)$ & $-53,5898384862$ & $-(200 \cdot \sqrt{ } 3-200)$ & $-146,410161514$ & 30 & 30 \\
\hline 4 & $-(100 \cdot \sqrt{ } 3-100)$ & $-73,2050807569$ & $-(300-100 \cdot \sqrt{ } 3)$ & $-126,794919243$ & $(80-25 \cdot \sqrt{ } 3)$ & 36,698729811 \\
\hline 5 & $-(100 \cdot \sqrt{3})$ & $-173,2050807569$ & -100 & -100 & 55 & 55 \\
\hline 6 & $-(82 \cdot \sqrt{ } 3+18)$ & $-160,0281662206$ & $-(82-18 \cdot \sqrt{ } 3)$ & $-50,823085464$ & 64 & 64 \\
\hline 7 & $-(148 \cdot \sqrt{ } 3-26)$ & $-230,3435195202$ & $-(26 \cdot \sqrt{3}+148)$ & $-193,033320997$ & 42 & 42 \\
\hline 8 & $-(146 \cdot \sqrt{ } 3+34)$ & $-286,8794179051$ & $-(146-34 \cdot \sqrt{ } 3)$ & $-87,110272543$ & -38 & -38 \\
\hline 9 & $-(130 \cdot \sqrt{3}+50)$ & $-275,1666049840$ & $-(130-50 \cdot \sqrt{ } 3)$ & $-43,397459622$ & -30 & -30 \\
\hline 10 & $-(500-200 \cdot \sqrt{ } 3)$ & $-153,5898384862$ & $(200-100 \cdot \sqrt{3})$ & 26,794919243 & -30 & -30 \\
\hline 11 & $-(200 \cdot \sqrt{ } 3-200)$ & $-146,4101615138$ & 0 & 0 & $-(80-25 \cdot \sqrt{3})$ & $-36,698729811$ \\
\hline 12 & $-(100 \cdot \sqrt{3})$ & $-173,2050807569$ & -100 & -100 & -55 & -55 \\
\hline 13 & $-(82 \cdot \sqrt{ } 3-18)$ & $-124,0281662206$ & $-(18 \cdot \sqrt{3}+82)$ & $-113,176914536$ & -64 & -64 \\
\hline 14 & $-(148 \cdot \sqrt{3}+26)$ & $-282,3435195202$ & $-(148-26 \cdot \sqrt{ } 3)$ & $-102,966679003$ & -42 & -42 \\
\hline 15 & $-(142 \cdot \sqrt{ } 3+22)$ & $-267,9512146748$ & $(142-22 \cdot \sqrt{ } 3)$ & 103,894882234 & -30 & -30 \\
\hline 16 & $-(142 \cdot \sqrt{3}+38)$ & $-283,9512146748$ & $(142-38 \cdot \sqrt{ } 3)$ & 76,182069312 & -30 & -30 \\
\hline 17 & $-(180 \cdot \sqrt{3})$ & $-311,7691453624$ & 180 & 180 & -106 & -106 \\
\hline 18 & $-(146 \cdot \sqrt{ } 3-34)$ & $-218,8794179051$ & $(34 \cdot \sqrt{ } 3+146)$ & 204,889727457 & -38 & -38 \\
\hline 19 & $-(148 \cdot \sqrt{ } 3-26)$ & $-230,3435195202$ & $(26 \cdot \sqrt{ } 3+148)$ & 193,033320997 & -42 & -42 \\
\hline 20 & $-(127 \cdot \sqrt{ } 3-12)$ & $-207,9704525612$ & $(12 \cdot \sqrt{3}+127)$ & 147,784609691 & 0 & 0 \\
\hline 21 & $-(127 \cdot \sqrt{3}+12)$ & $-231,9704525612$ & $(127-12 \cdot \sqrt{ } 3)$ & 106,215390309 & 0 & 0 \\
\hline 22 & $-(142 \cdot \sqrt{3}-22)$ & $-223,9512146748$ & $(22 \cdot \sqrt{ } 3+142)$ & 180,105117767 & 30 & 30 \\
\hline 23 & $-(142 \cdot \sqrt{ } 3-38)$ & $-207,9512146748$ & $(38 \cdot \sqrt{ } 3+142)$ & 207,817930688 & 30 & 30 \\
\hline 24 & $-(180 \cdot \sqrt{3})$ & $-311,7691453624$ & 180 & 180 & 106 & 106 \\
\hline 25 & $-(146 \cdot \sqrt{ } 3+34)$ & $-286,8794179051$ & $(146-34 \cdot \sqrt{ } 3)$ & 87,110272543 & 38 & 38 \\
\hline 26 & $-(148 \cdot \sqrt{ } 3+26)$ & $-282,3435195202$ & $(148-26 \cdot \sqrt{3})$ & 102,966679003 & 42 & 42 \\
\hline 27 & $-(100 \cdot \sqrt{3})$ & $-173,2050807569$ & 100 & 100 & -55 & -55 \\
\hline 28 & $-(100 \cdot \sqrt{ } 3)$ & $-173,2050807569$ & 100 & 100 & 55 & 55 \\
\hline 29 & $-(200 \cdot \sqrt{ } 3-200)$ & $-146,4101615138$ & 0 & 0 & $(80-25 \cdot \sqrt{ } 3)$ & 36,698729811 \\
\hline 30 & $-(82 \cdot \sqrt{ } 3+18)$ & $-160,0281662206$ & $(82-18 \cdot \sqrt{ } 3)$ & 50,823085464 & -30 & -30 \\
\hline 31 & $-(82 \cdot \sqrt{ } 3+18)$ & $-160,0281662206$ & $(82-18 \cdot \sqrt{ } 3)$ & 50,823085464 & -64 & -64 \\
\hline 32 & $-(130 \cdot \sqrt{ } 3+50)$ & $-275,1666049840$ & $(130-50 \cdot \sqrt{ } 3)$ & 43,397459622 & 30 & 30 \\
\hline 33 & $-(360 \cdot \sqrt{ } 3-360)$ & $-263,5382907248$ & 0 & 0 & -30 & -30 \\
\hline 34 & $-(360 \cdot \sqrt{ } 3-360)$ & $-263,5382907248$ & 0 & 0 & 30 & 30 \\
\hline 35 & $-(82 \cdot \sqrt{ } 3-18)$ & $-124,0281662206$ & $(18 \cdot \sqrt{ } 3+82)$ & 113,176914536 & 30 & 30 \\
\hline 36 & $-(82 \cdot \sqrt{ } 3-18)$ & $-124,0281662206$ & $(18 \cdot \sqrt{3}+82)$ & 113,176914536 & 64 & 64 \\
\hline 37 & $-(100 \cdot \sqrt{ } 3-100)$ & $-73,2050807569$ & $(300-100 \cdot \sqrt{ } 3)$ & 126,794919243 & $-(80-25 \cdot \sqrt{3})$ & $-36,698729811$ \\
\hline 38 & $-(100 \cdot \sqrt{ } 3-100)$ & $-73,2050807569$ & $(300-100 \cdot \sqrt{ } 3)$ & 126,794919243 & $(80-25 \cdot \sqrt{ } 3)$ & 36,698729811 \\
\hline 39 & -100 & -100 & $(300 \cdot \sqrt{ } 3-400)$ & 119,615242271 & 30 & 30 \\
\hline 40 & $-(142 \cdot \sqrt{ } 3-38)$ & $-207,9512146748$ & $-(38 \cdot \sqrt{3}+142)$ & $-207,817930688$ & -30 & -30 \\
\hline 41 & $-(142 \cdot \sqrt{ } 3-22)$ & $-223,9512146748$ & $-(22 \cdot \sqrt{ } 3+142)$ & $-180,105117767$ & -30 & -30 \\
\hline 42 & $-(109 \cdot \sqrt{ } 3)$ & $-188,7935380250$ & -109 & -109 & -30 & -30 \\
\hline 43 & $-(82 \cdot \sqrt{ } 3-18)$ & $-124,0281662206$ & $-(18 \cdot \sqrt{3}+82)$ & $-113,176914536$ & -30 & -30 \\
\hline 44 & -100 & -100 & $-(300 \cdot \sqrt{ } 3-400)$ & $-119,615242271$ & -30 & -30 \\
\hline 45 & -100 & -100 & -260 & -260 & -30 & -30 \\
\hline 46 & $-(180 \cdot \sqrt{ } 3-180)$ & $-131,7691453624$ & $-(540-180 \cdot \sqrt{ } 3)$ & $-228,230854638$ & -30 & -30 \\
\hline 47 & $-(109 \cdot \sqrt{3})$ & $-188,7935380250$ & 109 & 109 & -30 & -30 \\
\hline 48 & 0 & 0 & 360 & 360 & 106 & 106 \\
\hline 49 & 0 & 0 & 360 & 360 & -106 & -106 \\
\hline 50 & -76 & -76 & 284 & 284 & -30 & -30 \\
\hline 51 & $-(180 \cdot \sqrt{ } 3-180)$ & $-131,7691453624$ & $(540-180 \cdot \sqrt{ } 3)$ & 228,230854638 & -30 & -30 \\
\hline 52 & $-(180 \cdot \sqrt{ } 3-180)$ & $-131,7691453624$ & $(540-180 \cdot \sqrt{ } 3)$ & 228,230854638 & 30 & 30 \\
\hline
\end{tabular}




\begin{tabular}{|c|c|c|c|c|c|c|}
\hline \multirow{2}{*}{$\begin{array}{l}\text { № } \\
53\end{array}$} & \multicolumn{2}{|c|}{ Coordinates $\mathrm{X}$} & \multicolumn{2}{|c|}{ Coordinates $\mathrm{Y}$} & \multicolumn{2}{|c|}{ Coordinates $\mathrm{Z}$} \\
\hline & -100 & -100 & 260 & 260 & 30 & 30 \\
\hline 54 & -68 & -68 & 292 & 292 & 38 & 38 \\
\hline 55 & 44 & 44 & 284 & 284 & 30 & 30 \\
\hline 56 & 76 & 76 & 284 & 284 & 30 & 30 \\
\hline 57 & -52 & -52 & 296 & 296 & 42 & 42 \\
\hline 58 & -24 & -24 & 254 & 254 & 0 & 0 \\
\hline 59 & 24 & 24 & 254 & 254 & 0 & 0 \\
\hline 60 & $-(180 \cdot \sqrt{ } 3-180)$ & $-131,7691453624$ & $-(540-180 \cdot \sqrt{ } 3)$ & $-228,230854638$ & 30 & 30 \\
\hline 61 & -76 & -76 & -284 & -284 & 30 & 30 \\
\hline 62 & -44 & -44 & -284 & -284 & 30 & 30 \\
\hline 63 & 0 & 0 & -218 & -218 & 30 & 30 \\
\hline 64 & -36 & -36 & -164 & -164 & 30 & 30 \\
\hline 65 & $-(109 \cdot \sqrt{3})$ & $-188,7935380250$ & -109 & -109 & 30 & 30 \\
\hline 66 & $-(82 \cdot \sqrt{ } 3+18)$ & $-160,0281662206$ & $-(82-18 \cdot \sqrt{ } 3)$ & $-50,823085464$ & 30 & 30 \\
\hline 67 & $-(500-200 \cdot \sqrt{ } 3)$ & $-153,5898384862$ & $-(200-100 \cdot \sqrt{ } 3)$ & $-26,794919243$ & 30 & 30 \\
\hline 68 & $-(142 \cdot \sqrt{ } 3+38)$ & $-283,9512146748$ & $-(142-38 \cdot \sqrt{ } 3)$ & $-76,182069312$ & 30 & 30 \\
\hline 69 & $-(142 \cdot \sqrt{ } 3+22)$ & $-267,9512146748$ & $-(142-22 \cdot \sqrt{ } 3)$ & $-103,894882234$ & 30 & 30 \\
\hline 70 & 24 & 24 & -254 & -254 & 0 & 0 \\
\hline 71 & 52 & 52 & -296 & -296 & 42 & 42 \\
\hline 72 & -36 & -36 & -164 & -164 & 64 & 64 \\
\hline 73 & 0 & 0 & -218 & -218 & -30 & -30 \\
\hline 74 & 44 & 44 & -284 & -284 & -30 & -30 \\
\hline 75 & 100 & 100 & 260 & 260 & -30 & -30 \\
\hline 76 & 68 & 68 & 292 & 292 & -38 & -38 \\
\hline 77 & $(180 \cdot \sqrt{3}-180)$ & 131,7691453624 & $(540-180 \cdot \sqrt{ } 3)$ & 228,230854638 & 30 & 30 \\
\hline 78 & $(180 \cdot \sqrt{ } 3-180)$ & 131,7691453624 & $(540-180 \cdot \sqrt{ } 3)$ & 228,230854638 & -30 & -30 \\
\hline 79 & 0 & 0 & -360 & -360 & 106 & 106 \\
\hline 80 & 68 & 68 & -292 & -292 & 38 & 38 \\
\hline 81 & -24 & -24 & -254 & -254 & 0 & 0 \\
\hline 82 & $-(100 \cdot \sqrt{ } 3-100)$ & $-73,2050807569$ & $-(300-100 \cdot \sqrt{ } 3)$ & $-126,794919243$ & $-(80-25 \cdot \sqrt{ } 3)$ & $-36,698729811$ \\
\hline 83 & 76 & 76 & -284 & -284 & -30 & -30 \\
\hline 84 & 0 & 0 & -360 & -360 & -106 & -106 \\
\hline 85 & -68 & -68 & -292 & -292 & -38 & -38 \\
\hline 86 & -52 & -52 & -296 & -296 & -42 & -42 \\
\hline 87 & 36 & 36 & 164 & 164 & 30 & 30 \\
\hline 88 & 36 & 36 & 164 & 164 & 64 & 64 \\
\hline 89 & 0 & 0 & 200 & 200 & 55 & 55 \\
\hline 90 & 0 & 0 & 200 & 200 & -55 & -55 \\
\hline 91 & $(100 \cdot \sqrt{ } 3-100)$ & 73,2050807569 & $(300-100 \cdot \sqrt{ } 3)$ & 126,794919243 & $-(80-25 \cdot \sqrt{ } 3)$ & $-36,698729811$ \\
\hline 92 & $(100 \cdot \sqrt{3}-100)$ & 73,2050807569 & $(300-100 \cdot \sqrt{ } 3)$ & 126,794919243 & $(80-25 \cdot \sqrt{ } 3)$ & 36,698729811 \\
\hline 93 & $(400-200 \cdot \sqrt{3})$ & 53,5898384862 & $(200 \cdot \sqrt{3}-200)$ & 146,410161514 & 30 & 30 \\
\hline 94 & $(127 \cdot \sqrt{ } 3-12)$ & 207,9704525612 & $-(12 \cdot \sqrt{3}+127)$ & $-147,784609691$ & 0 & 0 \\
\hline 95 & $(148 \cdot \sqrt{ } 3-26)$ & 230,3435195202 & $-(26 \cdot \sqrt{ } 3+148)$ & $-193,033320997$ & -42 & -42 \\
\hline 96 & $(82 \cdot \sqrt{ } 3+18)$ & 160,0281662206 & $-(82-18 \cdot \sqrt{ } 3)$ & $-50,823085464$ & -64 & -64 \\
\hline 97 & $(82 \cdot \sqrt{ } 3+18)$ & 160,0281662206 & $-(82-18 \cdot \sqrt{ } 3)$ & $-50,823085464$ & -30 & -30 \\
\hline 98 & $(109 \cdot \sqrt{ } 3)$ & 188,7935380250 & -109 & -109 & -30 & -30 \\
\hline 99 & $(109 \cdot \sqrt{ } 3)$ & 188,7935380250 & -109 & -109 & 30 & 30 \\
\hline 100 & $(142 \cdot \sqrt{3}-22)$ & 223,9512146748 & $-(22 \cdot \sqrt{3}+142)$ & $-180,105117767$ & 30 & 30 \\
\hline 101 & $(180 \cdot \sqrt{ } 3)$ & 311,7691453624 & 180 & 180 & 106 & 106 \\
\hline 102 & $(180 \cdot \sqrt{ } 3)$ & 311,7691453624 & 180 & 180 & -106 & -106 \\
\hline 103 & $(142 \cdot \sqrt{ } 3-38)$ & 207,9512146748 & $(38 \cdot \sqrt{ } 3+142)$ & 207,817930688 & -30 & -30 \\
\hline 104 & $(130 \cdot \sqrt{ } 3-50)$ & 175,1666049840 & $(50 \cdot \sqrt{ } 3+130)$ & 216,602540378 & 30 & 30 \\
\hline 105 & $(146 \cdot \sqrt{ } 3-34)$ & 218,8794179051 & $(34 \cdot \sqrt{ } 3+146)$ & 204,889727457 & 38 & 38 \\
\hline 106 & $(100 \cdot \sqrt{ } 3)$ & 173,2050807569 & -100 & -100 & 55 & 55 \\
\hline 107 & $(100 \cdot \sqrt{ } 3)$ & 173,2050807569 & -100 & -100 & -55 & -55 \\
\hline 108 & $(100 \cdot \sqrt{ } 3-100)$ & 73,2050807569 & $-(300-100 \cdot \sqrt{ } 3)$ & $-126,794919243$ & $-(80-25 \cdot \sqrt{ } 3)$ & $-36,698729811$ \\
\hline 109 & $(100 \cdot \sqrt{ } 3-100)$ & 73,2050807569 & $-(300-100 \cdot \sqrt{ } 3)$ & $-126,794919243$ & $(80-25 \cdot \sqrt{ } 3)$ & 36,698729811 \\
\hline 110 & 100 & 100 & $-(300 \cdot \sqrt{3}-400)$ & $-119,615242271$ & 30 & 30 \\
\hline 111 & $(82 \cdot \sqrt{ } 3-18)$ & 124,0281662206 & $-(18 \cdot \sqrt{ } 3+82)$ & $-113,176914536$ & 30 & 30 \\
\hline 112 & $(82 \cdot \sqrt{ } 3-18)$ & 124,0281662206 & $-(18 \cdot \sqrt{ } 3+82)$ & $-113,176914536$ & 64 & 64 \\
\hline 113 & 100 & 100 & $(300 \cdot \sqrt{ } 3-400)$ & 119,615242271 & -30 & -30 \\
\hline
\end{tabular}




\begin{tabular}{|c|c|c|c|c|c|c|}
\hline \multirow{2}{*}{$\begin{array}{c}\text { № } \\
114\end{array}$} & \multicolumn{2}{|c|}{ Coordinates $\mathrm{X}$} & \multicolumn{2}{|c|}{ Coordinates $\mathrm{Y}$} & \multicolumn{2}{|c|}{ Coordinates Z } \\
\hline & $(82 \cdot \sqrt{ } 3-18)$ & 124,0281662206 & $(18 \cdot \sqrt{3}+82)$ & 113,176914536 & -30 & -30 \\
\hline 115 & $(82 \cdot \sqrt{3}-18)$ & 124,0281662206 & $(18 \cdot \sqrt{3}+82)$ & 113,176914536 & -64 & -64 \\
\hline 116 & $(100 \cdot \sqrt{ } 3)$ & 173,2050807569 & 100 & 100 & -55 & -55 \\
\hline 117 & $(100 \cdot \sqrt{ } 3)$ & 173,2050807569 & 100 & 100 & 55 & 55 \\
\hline 118 & $(142 \cdot \sqrt{ } 3+22)$ & 267,9512146748 & $(142-22 \cdot \sqrt{ } 3)$ & 103,894882234 & 30 & 30 \\
\hline 119 & $(142 \cdot \sqrt{3}+38)$ & 283,9512146748 & $(142-38 \cdot \sqrt{ } 3)$ & 76,182069312 & 30 & 30 \\
\hline 120 & $(148 \cdot \sqrt{ } 3-26)$ & 230,3435195202 & $(26 \cdot \sqrt{3}+148)$ & 193,033320997 & 42 & 42 \\
\hline 121 & $(127 \cdot \sqrt{ } 3-12)$ & 207,9704525612 & $(12 \cdot \sqrt{ } 3+127)$ & 147,784609691 & 0 & 0 \\
\hline 122 & $(127 \cdot \sqrt{3}+12)$ & 231,9704525612 & $(127-12 \cdot \sqrt{3})$ & 106,215390309 & 0 & 0 \\
\hline 123 & $(142 \cdot \sqrt{ } 3+22)$ & 267,9512146748 & $-(142-22 \cdot \sqrt{ } 3)$ & $-103,894882234$ & -30 & -30 \\
\hline 124 & $(142 \cdot \sqrt{ } 3+38)$ & 283,9512146748 & $-(142-38 \cdot \sqrt{ } 3)$ & $-76,182069312$ & -30 & -30 \\
\hline 125 & $(180 \cdot \sqrt{3})$ & 311,7691453624 & -180 & -180 & -106 & -106 \\
\hline 126 & $(146 \cdot \sqrt{ } 3-34)$ & 218,8794179051 & $-(34 \cdot \sqrt{ } 3+146)$ & $-204,889727457$ & -38 & -38 \\
\hline 127 & $(127 \cdot \sqrt{ } 3+12)$ & 231,9704525612 & $-(127-12 \cdot \sqrt{ } 3)$ & $-106,215390309$ & 0 & 0 \\
\hline 128 & $(148 \cdot \sqrt{3}+26)$ & 282,3435195202 & $-(148-26 \cdot \sqrt{ } 3)$ & $-102,966679003$ & 42 & 42 \\
\hline 129 & $(130 \cdot \sqrt{ } 3+50)$ & 275,1666049840 & $(130-50 \cdot \sqrt{ } 3)$ & 43,397459622 & -30 & -30 \\
\hline 130 & $(146 \cdot \sqrt{ } 3+34)$ & 286,8794179051 & $(146-34 \cdot \sqrt{ } 3)$ & 87,110272543 & -38 & -38 \\
\hline 131 & $(360 \cdot \sqrt{ } 3-360)$ & 263,5382907248 & 0 & 0 & 30 & 30 \\
\hline 132 & $(360 \cdot \sqrt{ } 3-360)$ & 263,5382907248 & 0 & 0 & -30 & -30 \\
\hline 133 & $(142 \cdot \sqrt{ } 3-38)$ & 207,9512146748 & $-(38 \cdot \sqrt{ } 3+142)$ & $-207,817930688$ & 30 & 30 \\
\hline 134 & $(180 \cdot \sqrt{ } 3)$ & 311,7691453624 & -180 & -180 & 106 & 106 \\
\hline 135 & $(146 \cdot \sqrt{ } 3+34)$ & 286,8794179051 & $-(146-34 \cdot \sqrt{ } 3)$ & $-87,110272543$ & 38 & 38 \\
\hline 136 & $(130 \cdot \sqrt{ } 3+50)$ & 275,1666049840 & $-(130-50 \cdot \sqrt{ } 3)$ & $-43,397459622$ & 30 & 30 \\
\hline 137 & $-(130 \cdot \sqrt{ } 3-50)$ & $-175,1666049840$ & $(50 \cdot \sqrt{ } 3+130)$ & 216,602540378 & -30 & -30 \\
\hline 138 & $-(400-200 \cdot \sqrt{ } 3)$ & $-53,5898384862$ & $(200 \cdot \sqrt{ } 3-200)$ & 146,410161514 & -30 & -30 \\
\hline 139 & 0 & 0 & -200 & -200 & 55 & 55 \\
\hline 140 & 0 & 0 & -200 & -200 & -55 & -55 \\
\hline 141 & $(400-200 \cdot \sqrt{ } 3)$ & 53,5898384862 & $-(200 \cdot \sqrt{ } 3-200)$ & $-146,410161514$ & -30 & -30 \\
\hline 142 & 36 & 36 & -164 & -164 & -30 & -30 \\
\hline 143 & 36 & 36 & -164 & -164 & -64 & -64 \\
\hline 144 & $-(109 \cdot \sqrt{ } 3)$ & $-188,7935380250$ & 109 & 109 & 30 & 30 \\
\hline 145 & -44 & -44 & 284 & 284 & -30 & -30 \\
\hline 146 & 0 & 0 & 218 & 218 & -30 & -30 \\
\hline 147 & -36 & -36 & 164 & 164 & -30 & -30 \\
\hline 148 & $(148 \cdot \sqrt{3}+26)$ & 282,3435195202 & $(148-26 \cdot \sqrt{ } 3)$ & 102,966679003 & -42 & -42 \\
\hline 149 & $(109 \cdot \sqrt{ } 3)$ & 188,7935380250 & 109 & 109 & -30 & -30 \\
\hline 150 & $(109 \cdot \sqrt{ } 3)$ & 188,7935380250 & 109 & 109 & 30 & 30 \\
\hline 151 & $(142 \cdot \sqrt{ } 3-22)$ & 223,9512146748 & $(22 \cdot \sqrt{ } 3+142)$ & 180,105117767 & -30 & -30 \\
\hline 152 & 52 & 52 & 296 & 296 & -42 & -42 \\
\hline 153 & $(82 \cdot \sqrt{ } 3+18)$ & 160,0281662206 & $(82-18 \cdot \sqrt{ } 3)$ & 50,823085464 & 64 & 64 \\
\hline 154 & $(82 \cdot \sqrt{3}+18)$ & 160,0281662206 & $(82-18 \cdot \sqrt{ } 3)$ & 50,823085464 & 30 & 30 \\
\hline 155 & -36 & -36 & 164 & 164 & -64 & -64 \\
\hline 156 & $(200 \cdot \sqrt{ } 3-200)$ & 146,4101615138 & 0 & 0 & $-(80-25 \cdot \sqrt{ } 3)$ & $-36,698729811$ \\
\hline 157 & $(200 \cdot \sqrt{ } 3-200)$ & 146,4101615138 & 0 & 0 & $(80-25 \cdot \sqrt{ } 3)$ & 36,698729811 \\
\hline 158 & $(500-200 \cdot \sqrt{ } 3)$ & 153,5898384862 & $(200-100 \cdot \sqrt{ } 3)$ & 26,794919243 & 30 & 30 \\
\hline 159 & $(130 \cdot \sqrt{3}-50)$ & 175,1666049840 & $-(50 \cdot \sqrt{3}+130)$ & $-216,602540378$ & -30 & -30 \\
\hline 160 & $(180 \cdot \sqrt{ } 3-180)$ & 131,7691453624 & $-(540-180 \cdot \sqrt{ } 3)$ & $-228,230854638$ & 30 & 30 \\
\hline 161 & $(180 \cdot \sqrt{ } 3-180)$ & 131,7691453624 & $-(540-180 \cdot \sqrt{ } 3)$ & $-228,230854638$ & -30 & -30 \\
\hline 162 & 100 & 100 & -260 & -260 & 30 & 30 \\
\hline 163 & $(500-200 \cdot \sqrt{ } 3)$ & 153,5898384862 & $-(200-100 \cdot \sqrt{ } 3)$ & $-26,794919243$ & -30 & -30 \\
\hline 164 & 0 & 0 & 218 & 218 & 30 & 30 \\
\hline 165 & $-(127 \cdot \sqrt{ } 3-12)$ & $-207,9704525612$ & $-(12 \cdot \sqrt{ } 3+127)$ & $-147,784609691$ & 0 & 0 \\
\hline 166 & $-(127 \cdot \sqrt{ } 3+12)$ & $-231,9704525612$ & $-(127-12 \cdot \sqrt{ } 3)$ & $-106,215390309$ & 0 & 0 \\
\hline 167 & $-(180 \cdot \sqrt{ } 3)$ & $-311,7691453624$ & -180 & -180 & 106 & 106 \\
\hline 168 & $-(180 \cdot \sqrt{ } 3)$ & $-311,7691453624$ & -180 & -180 & -106 & -106 \\
\hline
\end{tabular}


Coordinates of face vertexes $(7,3 ; 7)-\mathrm{V} 1$.

$\underline{\text { Table } 3}$

\begin{tabular}{|c|c|c|c|c|c|c|c|c|c|c|c|c|c|c|c|}
\hline \multirow{2}{*}{$\begin{array}{l}\text { Face } \\
\text { № }\end{array}$} & \multicolumn{7}{|c|}{ Vertex number from the table 2 . } & \multirow{2}{*}{$\begin{array}{c}\text { Face } \\
\text { № }\end{array}$} & \multicolumn{7}{|c|}{ Vertex number from the table 2 . } \\
\hline & 1 & 2 & 3 & 4 & 5 & 6 & 7 & & 1 & 2 & 3 & 4 & 5 & 6 & 7 \\
\hline 1 & 1 & 2 & 3 & 4 & 5 & 6 & 7 & 37 & 122 & 148 & 115 & 114 & 149 & 150 & 118 \\
\hline 2 & 8 & 9 & 10 & 11 & 12 & 13 & 14 & 38 & 151 & 103 & 102 & 130 & 148 & 122 & 121 \\
\hline 3 & 15 & 16 & 17 & 18 & 19 & 20 & 21 & 39 & 145 & 50 & 49 & 76 & 152 & 59 & 58 \\
\hline 4 & 22 & 23 & 24 & 25 & 26 & 21 & 20 & 40 & 121 & 120 & 153 & 154 & 150 & 149 & 151 \\
\hline 5 & 27 & 28 & 29 & 11 & 10 & 30 & 31 & 41 & 54 & 53 & 39 & 38 & 89 & 88 & 57 \\
\hline 6 & 32 & 25 & 24 & 17 & 16 & 33 & 34 & 42 & 76 & 75 & 113 & 91 & 90 & 155 & 152 \\
\hline 7 & 35 & 36 & 28 & 27 & 37 & 38 & 39 & 43 & 117 & 116 & 156 & 157 & 158 & 154 & 153 \\
\hline 8 & 40 & 41 & 42 & 43 & 44 & 45 & 46 & 44 & 159 & 126 & 125 & 134 & 133 & 160 & 161 \\
\hline 9 & 10 & 9 & 33 & 16 & 15 & 47 & 30 & 45 & 81 & 86 & 143 & 142 & 73 & 63 & 62 \\
\hline 10 & 48 & 49 & 50 & 51 & 52 & 53 & 54 & 46 & 79 & 84 & 83 & 161 & 160 & 162 & 80 \\
\hline 11 & 55 & 56 & 48 & 54 & 57 & 58 & 59 & 47 & 113 & 75 & 78 & 103 & 151 & 149 & 114 \\
\hline 12 & 3 & 2 & 60 & 61 & 62 & 63 & 64 & 48 & 105 & 104 & 93 & 92 & 117 & 153 & 120 \\
\hline 13 & 65 & 66 & 67 & 32 & 34 & 68 & 69 & 49 & 130 & 129 & 163 & 156 & 116 & 115 & 148 \\
\hline 14 & 70 & 71 & 72 & 64 & 63 & 73 & 74 & 50 & 97 & 96 & 107 & 106 & 157 & 156 & 163 \\
\hline 15 & 75 & 76 & 49 & 48 & 56 & 77 & 78 & 51 & 163 & 129 & 132 & 124 & 123 & 98 & 97 \\
\hline 16 & 62 & 61 & 79 & 80 & 71 & 70 & 81 & 52 & 164 & 87 & 93 & 104 & 77 & 56 & 55 \\
\hline 17 & 4 & 82 & 44 & 43 & 13 & 12 & 5 & 53 & 135 & 136 & 158 & 157 & 106 & 112 & 128 \\
\hline 18 & 74 & 83 & 84 & 85 & 86 & 81 & 70 & 54 & 126 & 159 & 141 & 108 & 107 & 96 & 95 \\
\hline 19 & 87 & 88 & 89 & 90 & 91 & 92 & 93 & 55 & 141 & 159 & 161 & 83 & 74 & 73 & 142 \\
\hline 20 & 94 & 95 & 96 & 97 & 98 & 99 & 100 & 56 & 150 & 154 & 158 & 136 & 131 & 119 & 118 \\
\hline 21 & 101 & 102 & 103 & 78 & 77 & 104 & 105 & 57 & 65 & 42 & 41 & 165 & 7 & 6 & 66 \\
\hline 22 & 106 & 107 & 108 & 109 & 110 & 111 & 112 & 58 & 7 & 165 & 166 & 69 & 68 & 167 & 1 \\
\hline 23 & 92 & 91 & 113 & 114 & 115 & 116 & 117 & 59 & 65 & 69 & 166 & 14 & 13 & 43 & 42 \\
\hline 24 & 118 & 119 & 101 & 105 & 120 & 121 & 122 & 60 & 14 & 166 & 165 & 41 & 40 & 168 & 8 \\
\hline 25 & 123 & 124 & 125 & 126 & 95 & 94 & 127 & 61 & 58 & 57 & 88 & 87 & 164 & 146 & 145 \\
\hline 26 & 127 & 128 & 112 & 111 & 99 & 98 & 123 & 62 & 59 & 152 & 155 & 147 & 146 & 164 & 55 \\
\hline 27 & 129 & 130 & 102 & 101 & 119 & 131 & 132 & 63 & 21 & 26 & 36 & 35 & 144 & 47 & 15 \\
\hline 28 & 100 & 133 & 134 & 135 & 128 & 127 & 94 & 64 & 20 & 19 & 31 & 30 & 47 & 144 & 22 \\
\hline 29 & 134 & 125 & 124 & 132 & 131 & 136 & 135 & 65 & 80 & 162 & 110 & 109 & 139 & 72 & 71 \\
\hline 30 & 25 & 32 & 67 & 29 & 28 & 36 & 26 & 66 & 133 & 100 & 99 & 111 & 110 & 162 & 160 \\
\hline 31 & 18 & 137 & 138 & 37 & 27 & 31 & 19 & 67 & 167 & 168 & 40 & 46 & 60 & 2 & 1 \\
\hline 32 & 45 & 85 & 84 & 79 & 61 & 60 & 46 & 68 & 11 & 29 & 67 & 66 & 6 & 5 & 12 \\
\hline 33 & 64 & 72 & 139 & 140 & 82 & 4 & 3 & 69 & 38 & 37 & 138 & 147 & 155 & 90 & 89 \\
\hline 34 & 140 & 139 & 109 & 108 & 141 & 142 & 143 & 70 & 168 & 167 & 68 & 34 & 33 & 9 & 8 \\
\hline 35 & 23 & 22 & 144 & 35 & 39 & 53 & 52 & 71 & 17 & 24 & 23 & 52 & 51 & 137 & 18 \\
\hline 36 & 138 & 137 & 51 & 50 & 145 & 146 & 147 & 72 & 85 & 45 & 44 & 82 & 140 & 143 & 86 \\
\hline
\end{tabular}




\section{Polyhedron $(7,3 ; 7)-\mathrm{V} 2$.}

The polyhedron also consists of 72 heptagonal faces and differs from the previous one in that all vertex coordinates are integer, since the angles between the segments are straight. But this reduces the number of symmetries.

The polyhedron has two horizontal and one vertical 2-fold figure axes.
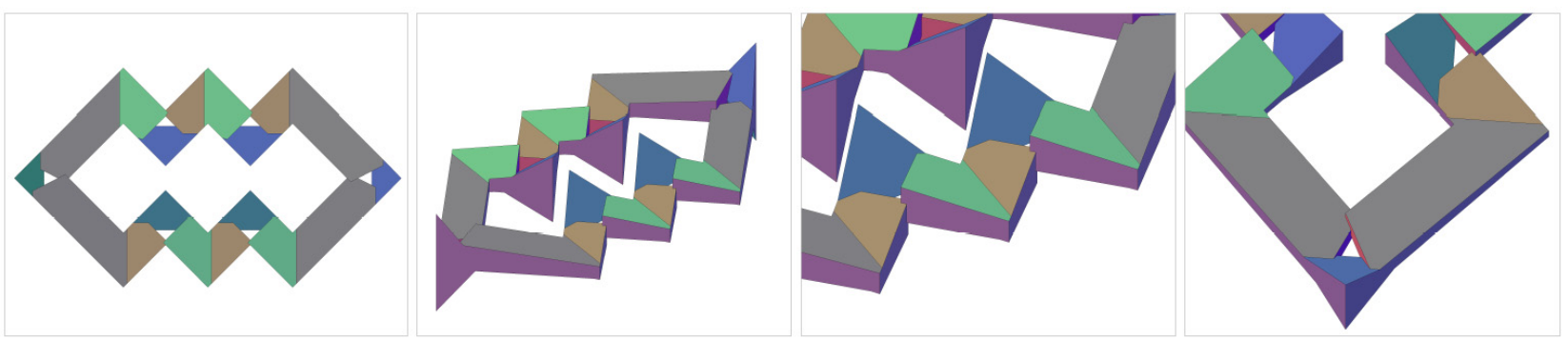

Fig. 6. General views $(7,3 ; 7)-\mathrm{V} 2$.
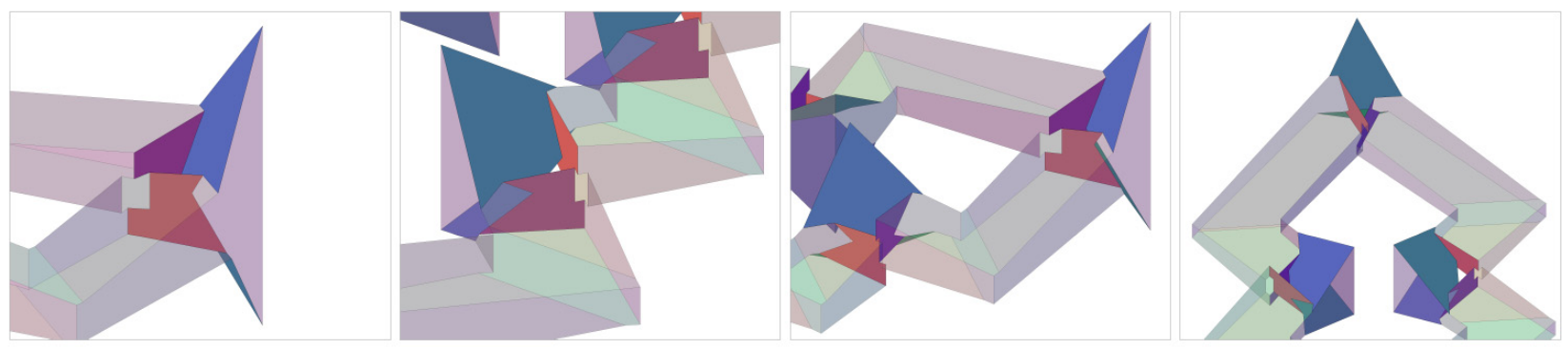

Fig. 7. Sections with enlarged scale and transparent faces $(7,3 ; 7)-\mathrm{V} 2$.

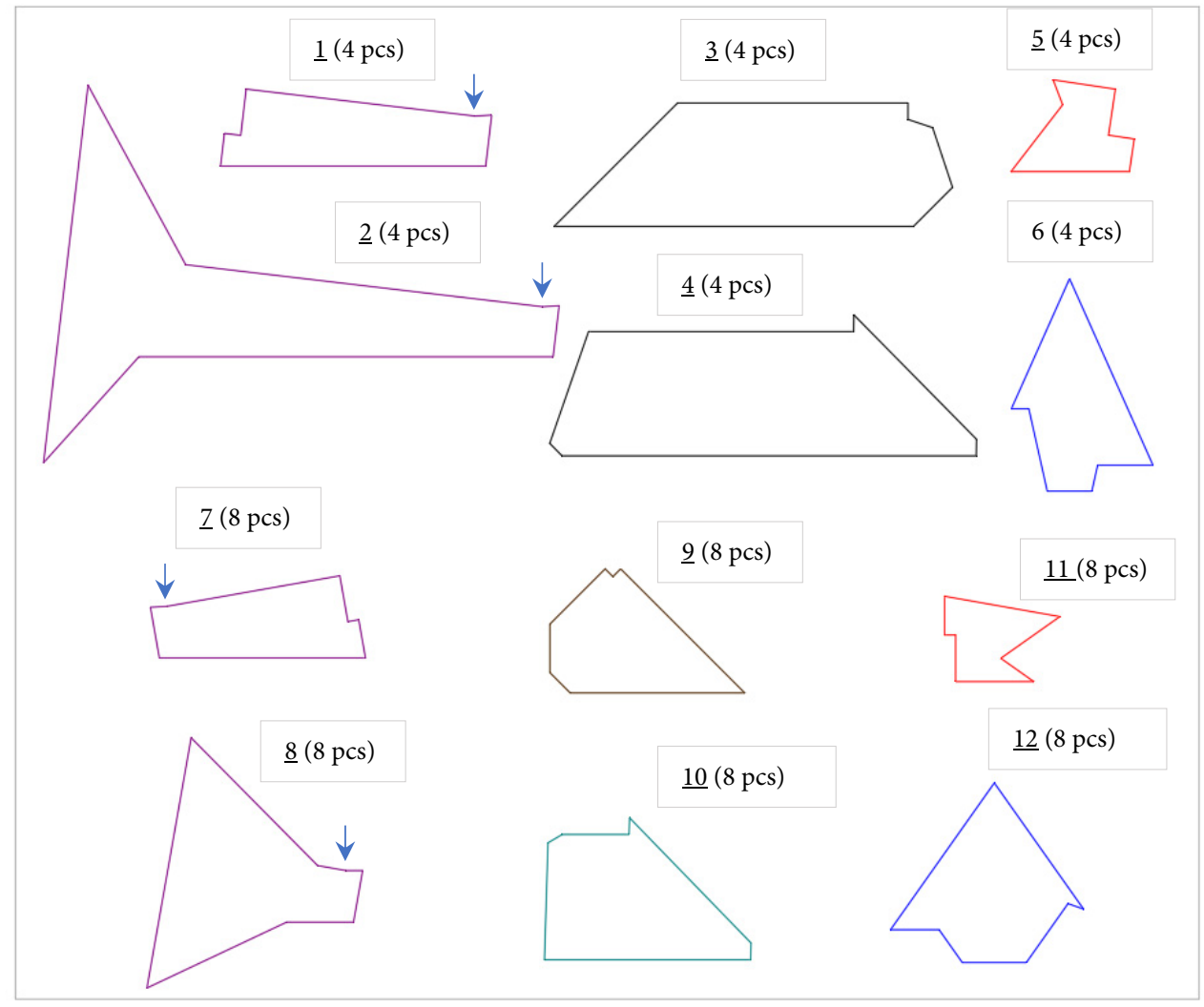

Fig. 8. Layout of faces on the plane $(7,3 ; 7)-\mathrm{V} 2$. 

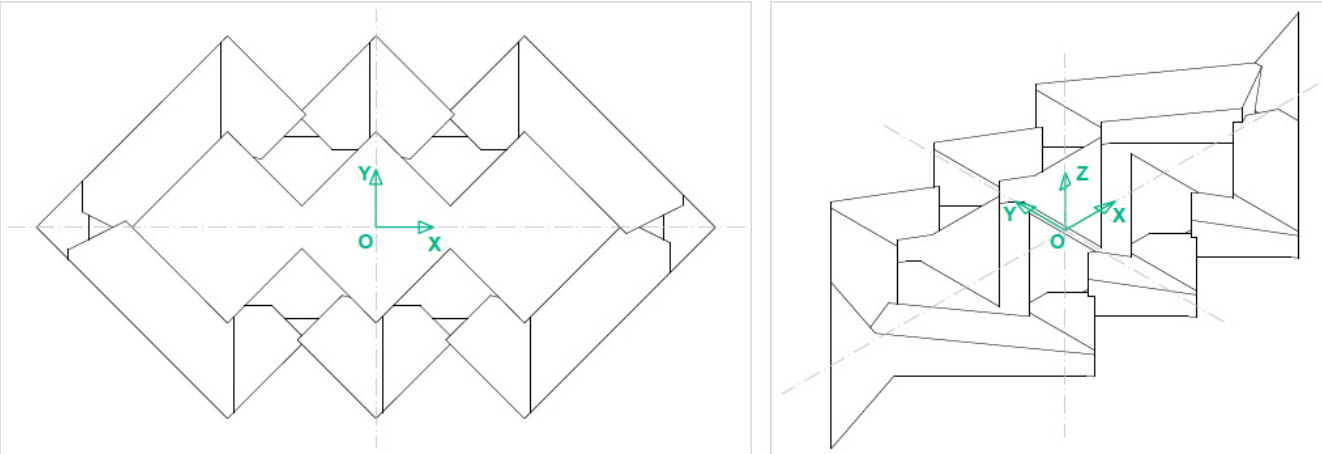

Fig. 9. Axis of symmetry $(7,3 ; 7)-V 2$.

Coordinates of polyhedron vertices $(7,3 ; 7)-\mathrm{V} 2$.

Table 4

\begin{tabular}{|c|c|c|c|c|c|c|c|c|c|c|c|c|c|c|c|}
\hline № & $\mathrm{X}$ & $\mathrm{Y}$ & $\mathrm{Z}$ & № & $\mathrm{X}$ & $\mathrm{Y}$ & $\mathrm{Z}$ & № & $\mathrm{X}$ & $\mathrm{Y}$ & $\mathrm{Z}$ & № & $\mathrm{X}$ & $\mathrm{Y}$ & $\mathrm{Z}$ \\
\hline 1 & -268 & 168 & -24 & 43 & 132 & -212 & 64 & 85 & 197 & -147 & -24 & 127 & -12 & -168 & -24 \\
\hline 2 & -280 & 180 & -26 & 44 & 268 & -348 & -24 & 86 & 247 & -147 & -24 & 128 & -12 & -348 & -24 \\
\hline 3 & -472 & -12 & -58 & 45 & 280 & -360 & -26 & 87 & 640 & 0 & -196 & 129 & -140 & -220 & -24 \\
\hline 4 & -580 & 42 & -76 & 46 & 132 & -212 & 24 & 88 & 472 & -12 & 24 & 130 & -132 & -212 & -24 \\
\hline 5 & -580 & 60 & -76 & 47 & 140 & -220 & 24 & 89 & 460 & 0 & 24 & 131 & -140 & -204 & -24 \\
\hline 6 & -280 & 360 & -26 & 48 & 140 & -220 & -24 & 90 & 496 & 0 & 24 & 132 & 148 & -212 & -64 \\
\hline 7 & -268 & 348 & -24 & 49 & 268 & 348 & 24 & 91 & 12 & 348 & -24 & 133 & 0 & -360 & -27 \\
\hline 8 & -554 & 29 & 24 & 50 & 280 & 360 & 26 & 92 & 0 & 360 & -27 & 134 & 0 & -180 & -27 \\
\hline 9 & -554 & 86 & 24 & 51 & 280 & 360 & -27 & 93 & 0 & 360 & 27 & 135 & -83 & 147 & 24 \\
\hline 10 & -640 & 0 & 196 & 52 & 132 & 212 & -64 & 94 & 148 & 212 & 64 & 136 & -140 & 204 & 24 \\
\hline 11 & -580 & -60 & 76 & 53 & 132 & 212 & -24 & 95 & 148 & 212 & 24 & 137 & -140 & 40 & 131 \\
\hline 12 & -580 & -42 & 76 & 54 & 140 & 220 & -24 & 96 & 268 & 168 & 24 & 138 & -232 & 132 & 39 \\
\hline 13 & -542 & -23 & 0 & 55 & 140 & 220 & 24 & 97 & 140 & 204 & 24 & 139 & 0 & 180 & 27 \\
\hline 14 & -542 & 23 & 0 & 56 & -140 & 220 & -24 & 98 & 197 & 147 & 24 & 140 & -12 & 168 & 24 \\
\hline 15 & -496 & 0 & -24 & 57 & -148 & 212 & -24 & 99 & 247 & 147 & 24 & 141 & -33 & 147 & 24 \\
\hline 16 & -472 & -12 & -24 & 58 & -140 & 204 & -24 & 100 & -64 & 128 & -43 & 142 & 140 & 40 & 131 \\
\hline 17 & -460 & 0 & -24 & 59 & -197 & 147 & -24 & 101 & -48 & 132 & -39 & 143 & 48 & 132 & 39 \\
\hline 18 & -292 & -168 & -24 & 60 & -247 & 147 & -24 & 102 & -140 & 40 & -131 & 144 & 64 & 128 & 43 \\
\hline 19 & -292 & -348 & -24 & 61 & -232 & -132 & -39 & 103 & -173 & 171 & 0 & 145 & -216 & 128 & 43 \\
\hline 20 & -554 & -86 & -24 & 62 & -216 & -128 & -43 & 104 & -107 & 171 & 0 & 146 & -12 & 348 & 24 \\
\hline 21 & -554 & -29 & -24 & 63 & -132 & -212 & -64 & 105 & 140 & 40 & -131 & 147 & -140 & 220 & 24 \\
\hline 22 & -640 & 0 & -196 & 64 & 268 & -168 & -24 & 106 & 33 & 147 & -24 & 148 & -132 & 212 & 24 \\
\hline 23 & -292 & 348 & 24 & 65 & 280 & -180 & -26 & 107 & 83 & 147 & -24 & 149 & -132 & 212 & 64 \\
\hline 24 & -280 & 360 & 27 & 66 & 472 & 12 & -58 & 108 & 107 & 171 & 0 & 150 & -140 & -220 & 24 \\
\hline 25 & -280 & -360 & -27 & 67 & 580 & -42 & -76 & 109 & 173 & 171 & 0 & 151 & -148 & -212 & 24 \\
\hline 26 & -280 & -360 & 26 & 68 & 580 & -60 & -76 & 110 & 216 & 128 & -43 & 152 & -140 & -204 & 24 \\
\hline 27 & -472 & 12 & 24 & 69 & 554 & -29 & 24 & 111 & 232 & 132 & -39 & 153 & -197 & -147 & 24 \\
\hline 28 & -460 & 0 & 24 & 70 & 554 & -86 & 24 & 112 & 12 & 168 & -24 & 154 & -247 & -147 & 24 \\
\hline 29 & -292 & 168 & 24 & 71 & 640 & 0 & 196 & 113 & 140 & 204 & -24 & 155 & 83 & -147 & 24 \\
\hline 30 & -496 & 0 & 24 & 72 & 580 & 60 & 76 & 114 & -148 & 212 & -64 & 156 & 140 & -204 & 24 \\
\hline 31 & -280 & 180 & 27 & 73 & 580 & 42 & 76 & 115 & 0 & 180 & -27 & 157 & 140 & -40 & 131 \\
\hline 32 & -472 & 12 & 58 & 74 & 542 & 23 & 0 & 116 & 280 & 180 & -27 & 158 & 0 & -180 & 27 \\
\hline 33 & -280 & -180 & 26 & 75 & 542 & -23 & 0 & 117 & 64 & -128 & -43 & 159 & 12 & -168 & 24 \\
\hline 34 & -280 & -180 & -27 & 76 & 496 & 0 & -24 & 118 & 48 & -132 & -39 & 160 & 33 & -147 & 24 \\
\hline 35 & -268 & -168 & 24 & 77 & 472 & 12 & -24 & 119 & 140 & -40 & -131 & 161 & -140 & -40 & 131 \\
\hline 36 & -268 & -348 & 24 & 78 & 460 & 0 & -24 & 120 & 173 & -171 & 0 & 162 & -48 & -132 & 39 \\
\hline 37 & 280 & -360 & 27 & 79 & 292 & 168 & -24 & 121 & 107 & -171 & 0 & 163 & 0 & -360 & 27 \\
\hline 38 & 292 & -348 & 24 & 80 & 292 & 348 & -24 & 122 & -140 & -40 & -131 & 164 & -148 & -212 & 64 \\
\hline 39 & 292 & -168 & 24 & 81 & 554 & 86 & -24 & 123 & -33 & -147 & -24 & 165 & -64 & -128 & 43 \\
\hline 40 & 280 & -180 & 27 & 82 & 554 & 29 & -24 & 124 & -83 & -147 & -24 & 166 & 12 & -348 & 24 \\
\hline 41 & 232 & -132 & 39 & 83 & 148 & -212 & -24 & 125 & -107 & -171 & 0 & 167 & 280 & 180 & 26 \\
\hline 42 & 216 & -128 & 43 & 84 & 140 & -204 & -24 & 126 & -173 & -171 & 0 & 168 & 472 & -12 & 58 \\
\hline
\end{tabular}


Coordinates of face vertexes $(7,3 ; 7)-\mathrm{V} 2$.

Table 5

\begin{tabular}{|c|c|c|c|c|c|c|c|c|c|c|c|c|c|c|c|}
\hline \multirow{2}{*}{$\begin{array}{c}\text { Face } \\
\text { № }\end{array}$} & \multicolumn{7}{|c|}{ Vertex number from the table 2.} & \multirow{2}{*}{$\begin{array}{c}\text { Face } \\
\text { № }\end{array}$} & \multicolumn{7}{|c|}{ Vertex number from the table 2.} \\
\hline & 1 & 2 & 3 & 4 & 1 & 2 & 3 & & 1 & 2 & 3 & 4 & 1 & 2 & 3 \\
\hline 1 & 1 & 2 & 3 & 4 & 5 & 6 & 7 & 37 & 118 & 117 & 132 & 133 & 128 & 127 & 134 \\
\hline 2 & 8 & 9 & 10 & 11 & 12 & 13 & 14 & 38 & 109 & 98 & 97 & 113 & 53 & 52 & 110 \\
\hline 3 & 15 & 16 & 17 & 18 & 19 & 20 & 21 & 39 & 104 & 135 & 136 & 58 & 57 & 114 & 100 \\
\hline 4 & 22 & 10 & 9 & 23 & 24 & 6 & 5 & 40 & 102 & 137 & 138 & 31 & 2 & 1 & 60 \\
\hline 5 & 25 & 26 & 11 & 10 & 22 & 20 & 19 & 41 & 115 & 139 & 140 & 141 & 137 & 102 & 101 \\
\hline 6 & 27 & 28 & 29 & 23 & 9 & 8 & 30 & 42 & 105 & 142 & 143 & 139 & 115 & 112 & 106 \\
\hline 7 & 4 & 14 & 13 & 21 & 20 & 22 & 5 & 43 & 144 & 143 & 142 & 99 & 98 & 109 & 108 \\
\hline 8 & 14 & 4 & 3 & 16 & 15 & 30 & 8 & 44 & 137 & 141 & 135 & 104 & 103 & 145 & 138 \\
\hline 9 & 29 & 28 & 17 & 16 & 3 & 2 & 31 & 45 & 135 & 141 & 140 & 146 & 147 & 148 & 136 \\
\hline 10 & 28 & 27 & 32 & 33 & 34 & 18 & 17 & 46 & 56 & 147 & 146 & 93 & 92 & 114 & 57 \\
\hline 11 & 13 & 12 & 32 & 27 & 30 & 15 & 21 & 47 & 143 & 144 & 94 & 93 & 146 & 140 & 139 \\
\hline 12 & 35 & 33 & 32 & 12 & 11 & 26 & 36 & 48 & 113 & 97 & 95 & 94 & 144 & 108 & 107 \\
\hline 13 & 37 & 38 & 39 & 40 & 41 & 42 & 43 & 49 & 58 & 136 & 148 & 149 & 145 & 103 & 59 \\
\hline 14 & 44 & 45 & 37 & 43 & 46 & 47 & 48 & 50 & 31 & 138 & 145 & 149 & 24 & 23 & 29 \\
\hline 15 & 49 & 50 & 51 & 52 & 53 & 54 & 55 & 51 & 6 & 24 & 149 & 148 & 147 & 56 & 7 \\
\hline 16 & 1 & 7 & 56 & 57 & 58 & 59 & 60 & 52 & 35 & 36 & 150 & 151 & 152 & 153 & 154 \\
\hline 17 & 34 & 61 & 62 & 63 & 25 & 19 & 18 & 53 & 126 & 153 & 152 & 131 & 130 & 63 & 62 \\
\hline 18 & 64 & 65 & 66 & 67 & 68 & 45 & 44 & 54 & 121 & 155 & 156 & 84 & 83 & 132 & 117 \\
\hline 19 & 69 & 70 & 71 & 72 & 73 & 74 & 75 & 55 & 39 & 89 & 78 & 77 & 66 & 65 & 40 \\
\hline 20 & 76 & 77 & 78 & 79 & 80 & 81 & 82 & 56 & 40 & 65 & 64 & 86 & 119 & 157 & 41 \\
\hline 21 & 64 & 44 & 48 & 83 & 84 & 85 & 86 & 57 & 134 & 158 & 159 & 160 & 157 & 119 & 118 \\
\hline 22 & 87 & 71 & 70 & 38 & 37 & 45 & 68 & 58 & 122 & 161 & 162 & 158 & 134 & 127 & 123 \\
\hline 23 & 51 & 50 & 72 & 71 & 87 & 81 & 80 & 59 & 129 & 150 & 36 & 26 & 25 & 63 & 130 \\
\hline 24 & 88 & 89 & 39 & 38 & 70 & 69 & 90 & 60 & 133 & 163 & 164 & 151 & 150 & 129 & 128 \\
\hline 25 & 67 & 75 & 74 & 82 & 81 & 87 & 68 & 61 & 165 & 162 & 161 & 154 & 153 & 126 & 125 \\
\hline 26 & 75 & 67 & 66 & 77 & 76 & 90 & 69 & 62 & 157 & 160 & 155 & 121 & 120 & 42 & 41 \\
\hline 27 & 55 & 54 & 91 & 92 & 93 & 94 & 95 & 63 & 34 & 33 & 35 & 154 & 161 & 122 & 61 \\
\hline 28 & 96 & 49 & 55 & 95 & 97 & 98 & 99 & 64 & 155 & 160 & 159 & 166 & 47 & 46 & 156 \\
\hline 29 & 100 & 101 & 102 & 60 & 59 & 103 & 104 & 65 & 48 & 47 & 166 & 163 & 133 & 132 & 83 \\
\hline 30 & 105 & 106 & 107 & 108 & 109 & 110 & 111 & 66 & 162 & 165 & 164 & 163 & 166 & 159 & 158 \\
\hline 31 & 107 & 106 & 112 & 91 & 54 & 53 & 113 & 67 & 131 & 152 & 151 & 164 & 165 & 125 & 124 \\
\hline 32 & 101 & 100 & 114 & 92 & 91 & 112 & 115 & 68 & 84 & 156 & 46 & 43 & 42 & 120 & 85 \\
\hline 33 & 51 & 80 & 79 & 116 & 111 & 110 & 52 & 69 & 116 & 167 & 96 & 99 & 142 & 105 & 111 \\
\hline 34 & 117 & 118 & 119 & 86 & 85 & 120 & 121 & 70 & 89 & 88 & 168 & 167 & 116 & 79 & 78 \\
\hline 35 & 122 & 123 & 124 & 125 & 126 & 62 & 61 & 71 & 74 & 73 & 168 & 88 & 90 & 76 & 82 \\
\hline 36 & 124 & 123 & 127 & 128 & 129 & 130 & 131 & 72 & 96 & 167 & 168 & 73 & 72 & 50 & 49 \\
\hline
\end{tabular}


Polyhedron $(7,3 ; 3)-\mathrm{V} 1$.

Characteristics of a polyhedron $(7,3 ; 3)-\mathrm{V} 1$ :

\begin{tabular}{|c|c|c|c|c|c|c|}
\hline Genus, $\boldsymbol{g}$ & Faces, $\boldsymbol{f}$ & Vertices, $\boldsymbol{v}$ & Edges, $\boldsymbol{e}$ & Angles, $\boldsymbol{p}$ & $\boldsymbol{\alpha}_{1}$ & $\boldsymbol{\alpha}_{2}$ \\
\hline 3 & 24 & 56 & 84 & 7 & 180 \\
\hline & & & \\
\hline & & & \\
\hline
\end{tabular}

Fig. 10. General views $(7,3 ; 3)-\mathrm{V} 1$.
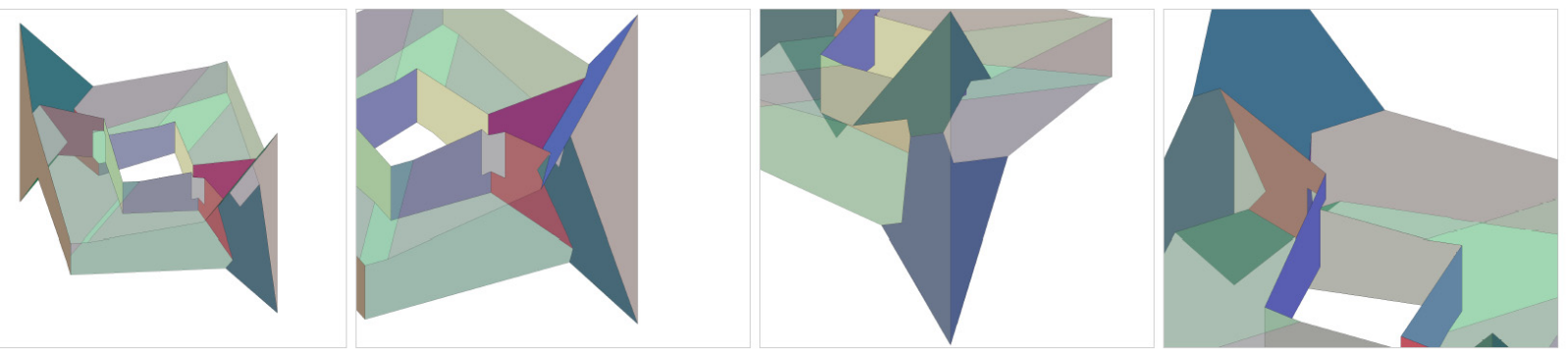

Fig. 11. Sections with enlarged scale and transparent faces $(7,3 ; 3)-\mathrm{V} 1$.

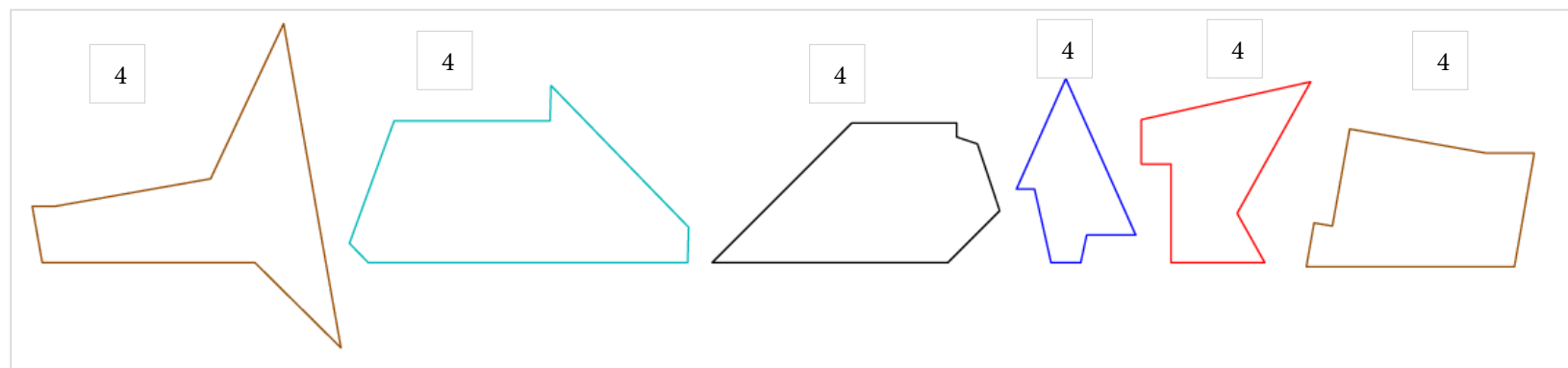

Fig. 12. Layout of faces on the plane $(7,3 ; 3)-\mathrm{V} 1$.

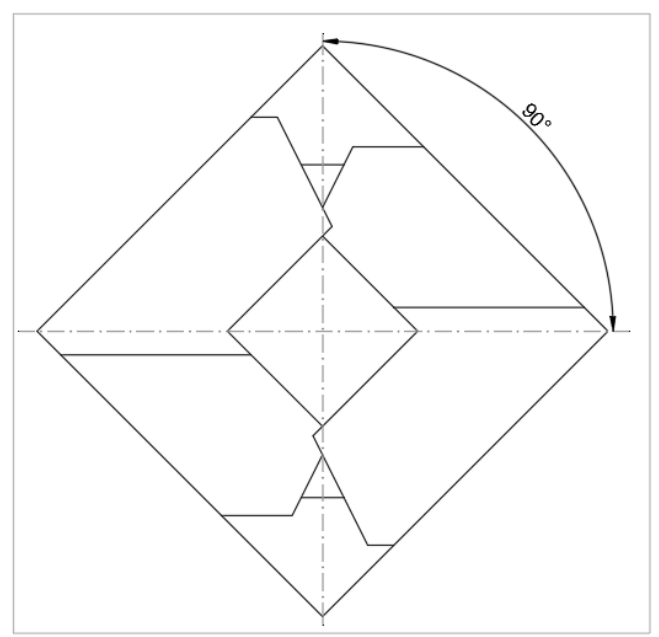

Fig. 13. Angles between segments. 
Polyhedron $(7,3 ; 4)-\mathrm{V} 1$.

Characteristics of a polyhedron $(7,3 ; 4)-\mathrm{V} 1$ :

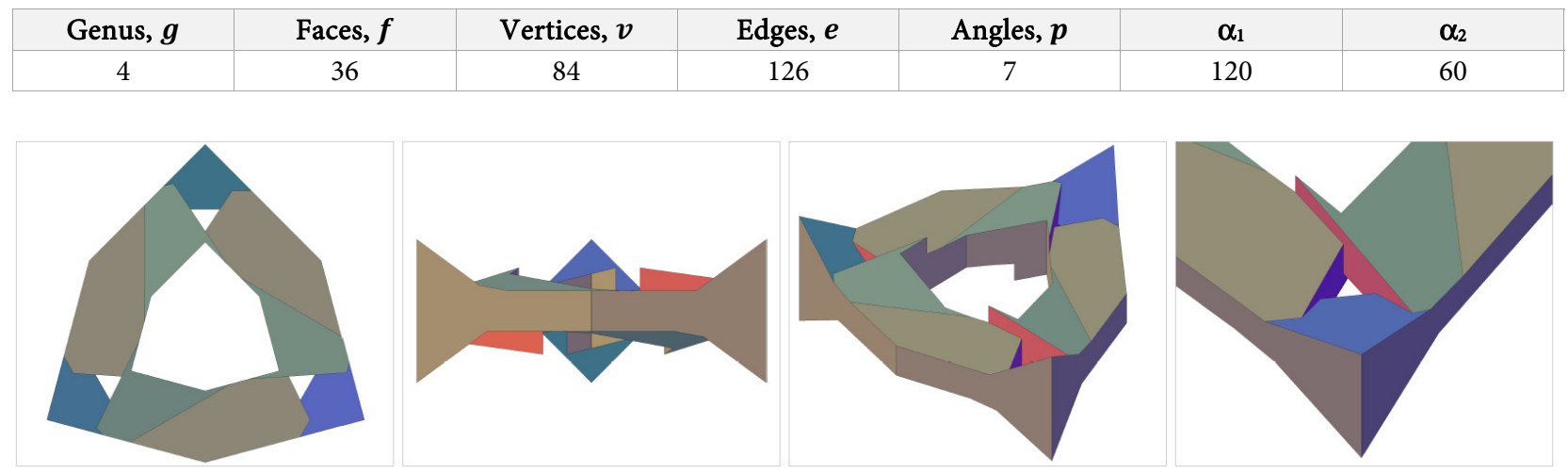

Fig. 14. General views $(7,3 ; 4)-\mathrm{V} 1$.
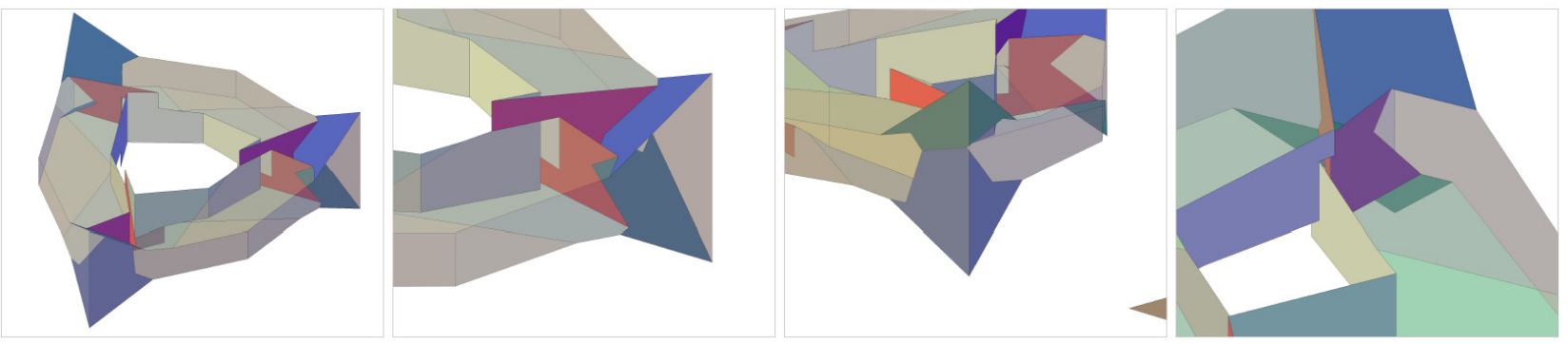

Fig. 15. Sections with enlarged scale and transparent faces $(7,3 ; 4)-\mathrm{V} 1$.

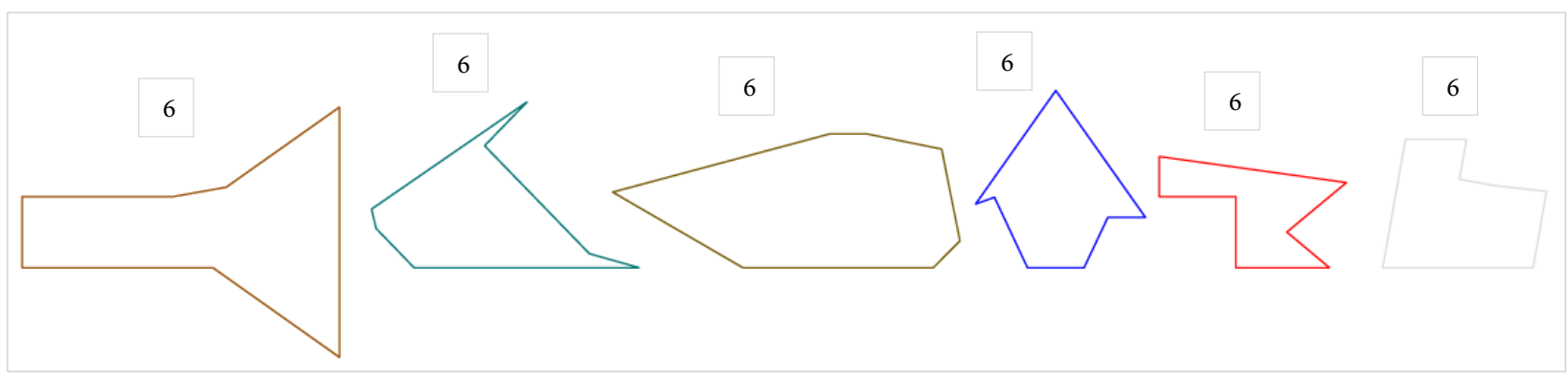

Fig. 16. Layout of faces on the plane $(7,3 ; 4)-\mathrm{V} 1$.

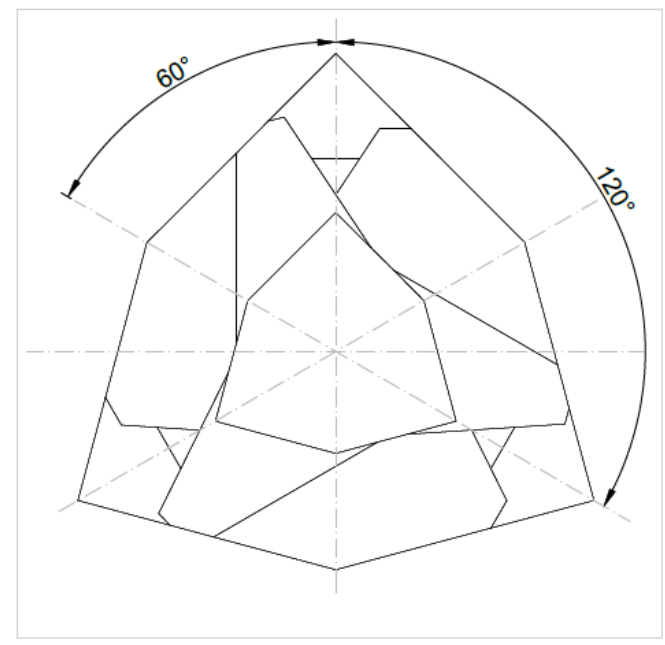

Fig. 17. Angles between segments. $(7,3 ; 4)-\mathrm{V} 1$. 
Polyhedron $(7,3 ; 5)-\mathrm{V} 1$.

Characteristics of a polyhedron $(7,3 ; 5)-\mathrm{V} 1$ :

\begin{tabular}{|c|c|c|c|c|c|c|}
\hline Genus, $\boldsymbol{g}$ & Faces, $\boldsymbol{f}$ & Vertices, $\boldsymbol{v}$ & Edges, $\boldsymbol{e}$ & Angles, $\boldsymbol{p}$ & $\boldsymbol{\alpha}_{1}$ & $\boldsymbol{\alpha}_{2}$ \\
\hline 5 & 48 & 112 & 168 & 7 & 90 & 45 \\
\hline
\end{tabular}
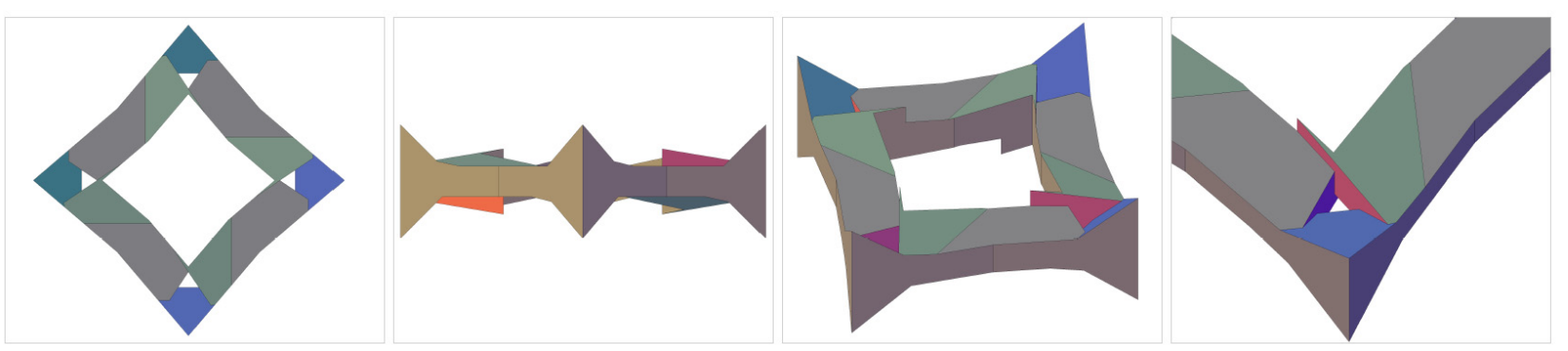

Fig. 18. General views $(7,3 ; 5)-\mathrm{V} 1$.
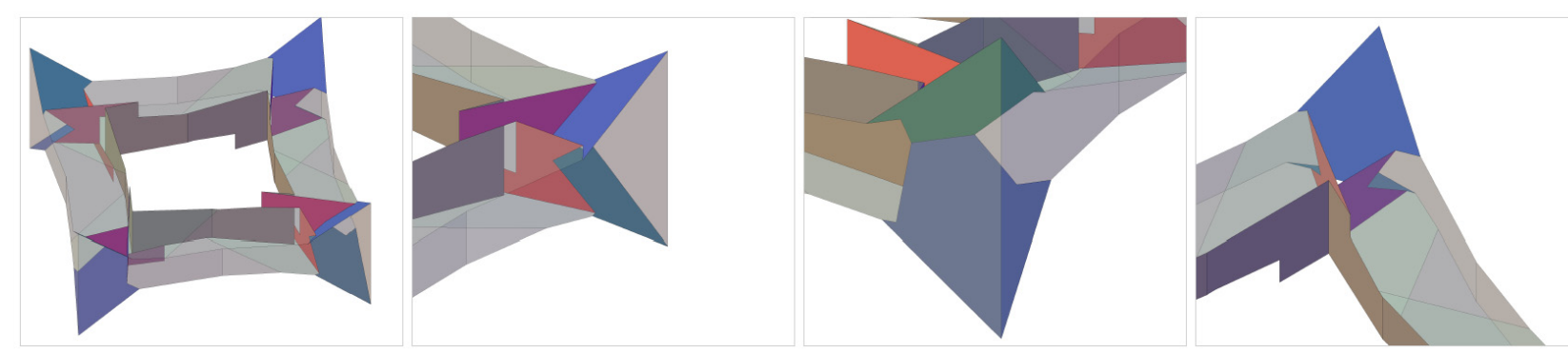

Fig. 19. Sections with enlarged scale and transparent faces $(7,3 ; 5)-V 1$.

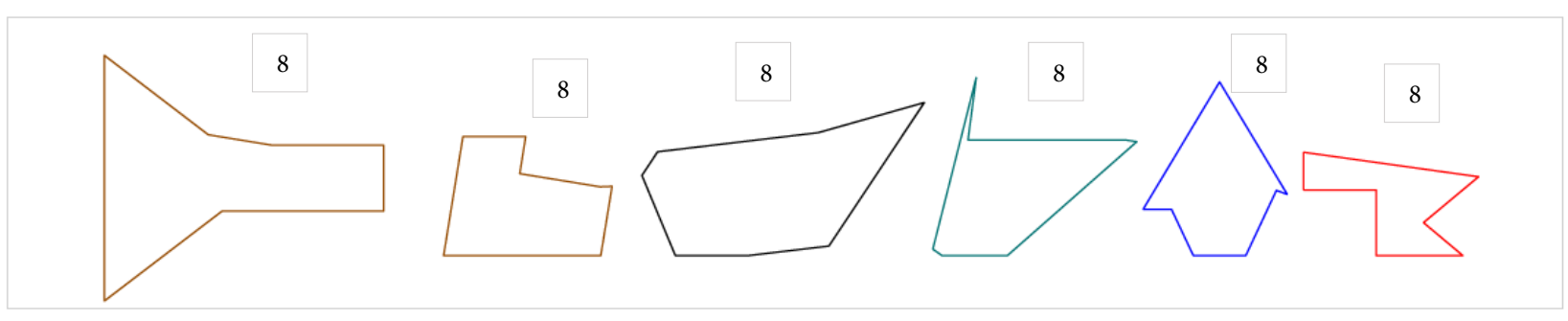

Fig. 20. Layout of faces on the plane $(7,3 ; 5)-\mathrm{V} 1$.

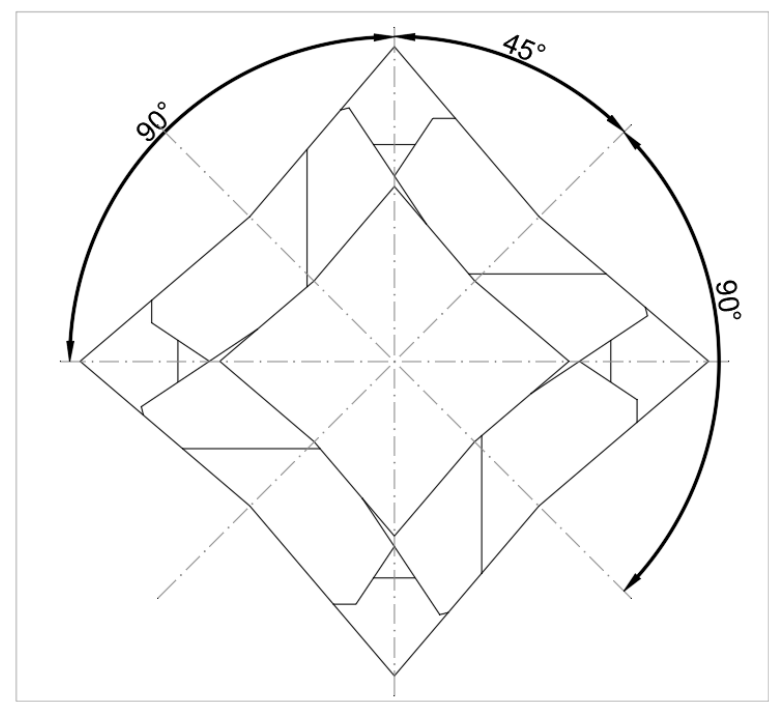

Fig. 21. Angles between segments $(7,3 ; 5)-\mathrm{V} 1$. 
Polyhedron $(7,3 ; 6)-\mathrm{V} 1$.

Characteristics of a polyhedron $(7,3 ; 6)-\mathrm{V} 1$ :

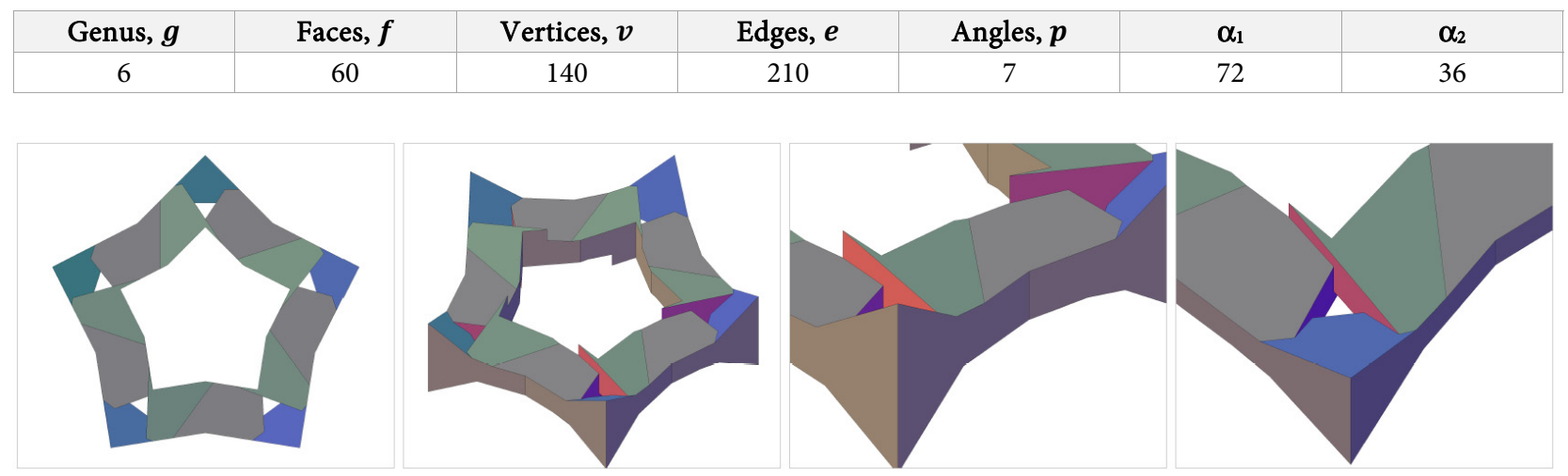

Fig. 22. General views $(7,3 ; 6)$-V1.
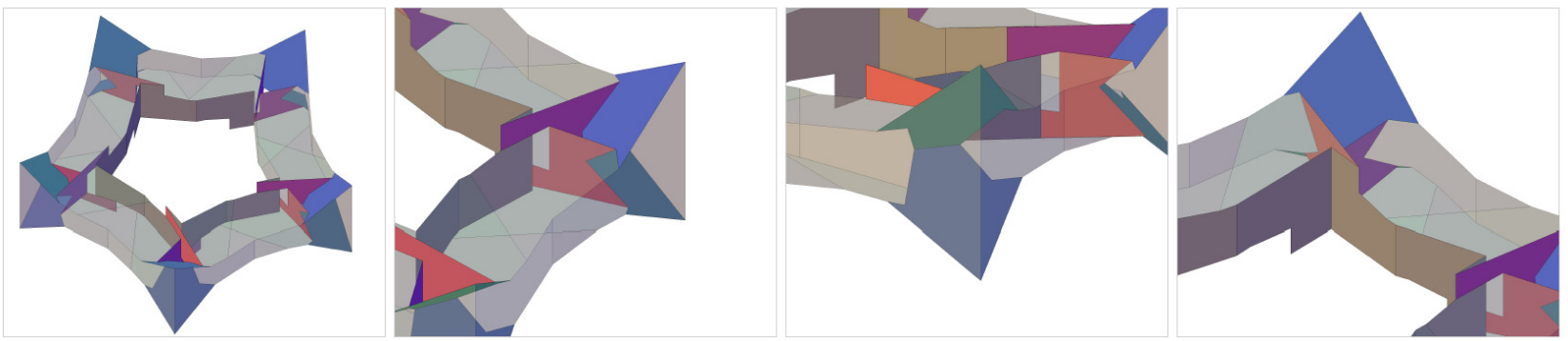

Fig. 23. Sections with enlarged scale and transparent faces $(7,3 ; 6)-\mathrm{V} 1$.

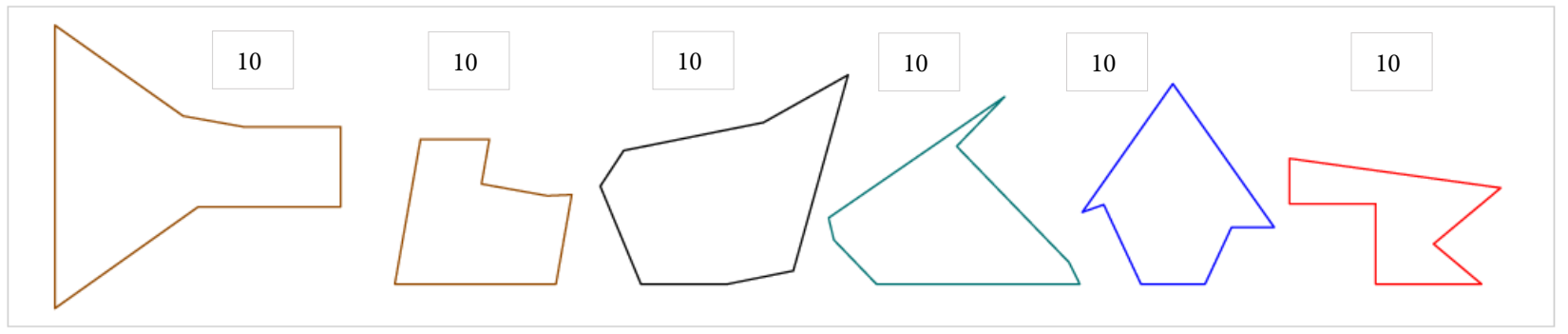

Fig. 24. Layout of faces on the plane $(7,3 ; 6)-\mathrm{V} 1$.

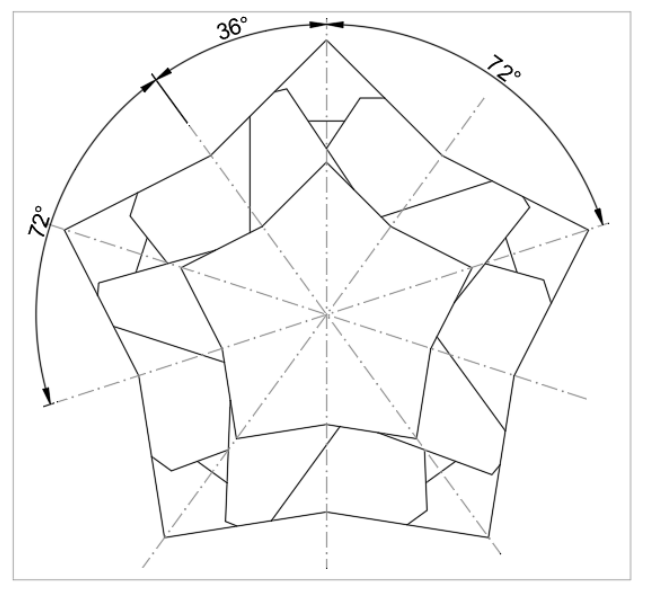

Fig. 25. Angles between segments $(7,3 ; 6)-\mathrm{V} 1$. 
Polyhedron $(7,3 ; 14)-\mathrm{V} 1$.

Characteristics of a polyhedron $(7,3 ; 14)-\mathrm{V} 1$ :

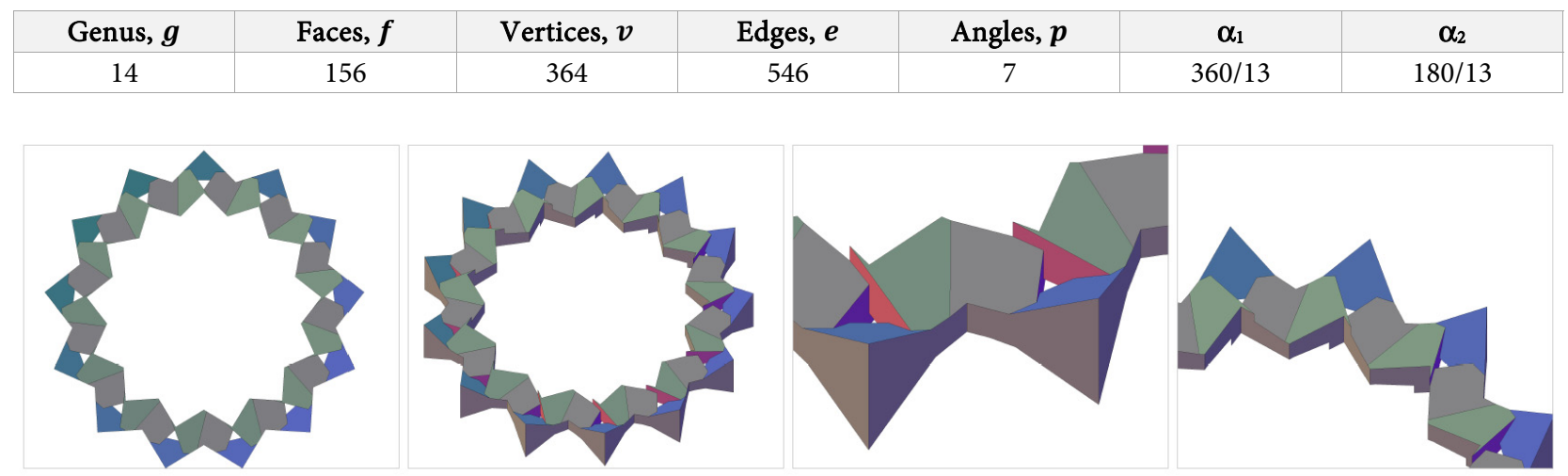

Fig. 26. General views $(7,3 ; 14)-\mathrm{V} 1$.
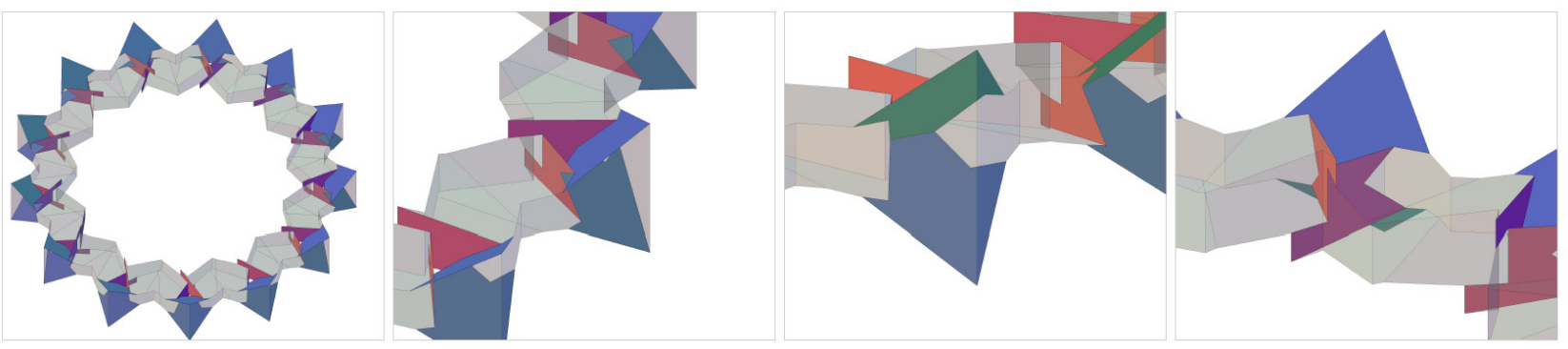

Fig. 27. Sections with enlarged scale and transparent faces $(7,3 ; 14)-\mathrm{V} 1$.
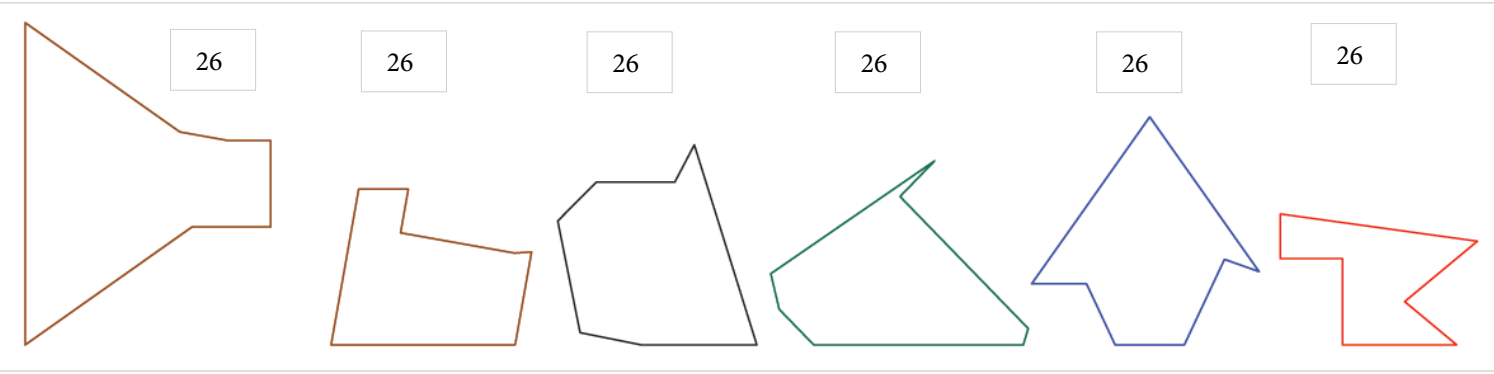

Fig. 28. Layout of faces on the plane $(7,3 ; 14)-\mathrm{V} 1$.

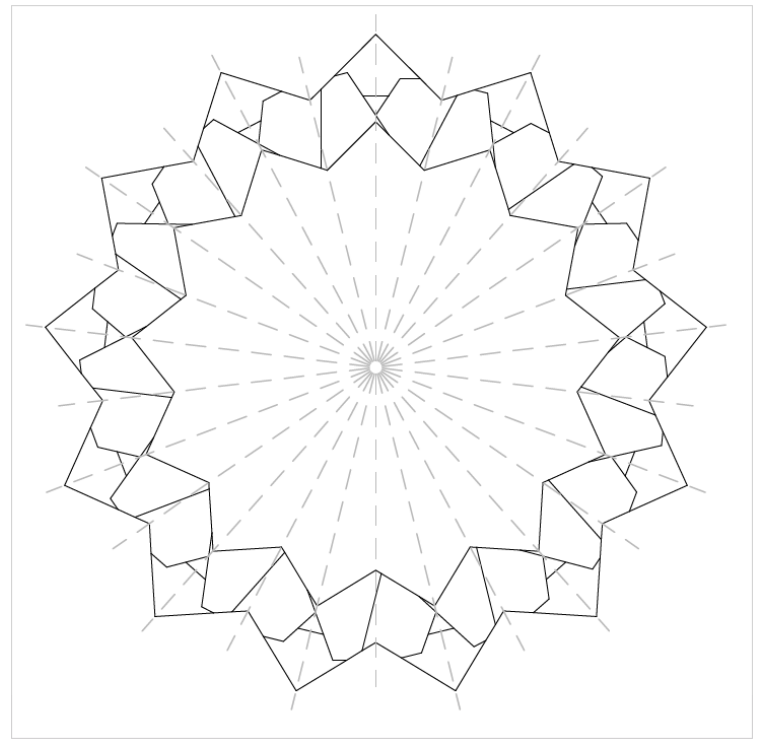

Fig. 29. Angles between segments $(7,3 ; 14)-\mathrm{V} 1$. 


\section{Conclusions}

This work allows us to conclude that locally regular toroidal polyhedra consisting of flat heptagonal faces are geometrically realizable in three-dimensional space. Based on the considered examples, one can notice the possibility to implement higher-genus polyhedra when changing the scale of faces and angles between segments. In this case, we can assume that an infinite realization is possible for this series.

\section{References}

[1] Ringel, Gerhard (1974). Map Color Theorem. New York/Berlin: Springer-Verlag.

[2] Szilassi, Lajos (1986), "Regular toroids", Structural Topology, 13: 69-80.

[3] J"org M. Wills. A locally regular heptagon-dodecahedron embedded in 3-space. Published in Eur. J. Comb. 2008.

[4] Branko Grünbaum, Lajos Szilassi. Geometric realizations of special toroidal complexes/ Contributions to Discrete Mathematics, Vol 4 No 1 (2009), pages 21-39. 2009. ISSN 1715-0868.

[5] David I. McCooey. Models of Locally Regular Heptagonal Dodecahedra. Pixelux Entertainment, Inc. Normandy Park, WA 98166, USA. Bridges 2010: Mathematics, Music, Art, Architecture, Culture.

[6] David I. McCooey. Higher Genus Toroidal Solids. http://dmccooey.com/polyhedra/HigherGenus.html

[7] J. Bokowski, A geometric realization without selfintersections does exist for Dyck's regular map, Discrete Comp. Geom. 6, 583-589.

[8] J"urgen Bokowski and Michael Cuntz. Hurwitz's regular map $(3,7)$ of genus 7 a polyhedral realization. July 18, 2017.

[9] F.H. Lutz, Enumeration and random realization of triangulated surfaces, Discrete Diff. Geom. (2007) (in press).

[10] J.M. Wills, Equivelar polyhedra, in: Ch. Davis, E. Ellers (Eds.), Proc. Coxeter Legacy, Toronto, 2004.

[11] G"unter M. Ziegler. Polyhedral surfaces of high genus. 2004. arXiv:math/0412093 [math.MG].

[12] J.M. Wills. On Polyhedra with Transitivity Properties. Discrete \& Computational Geometry volume 1, pages195-199(1986).

[13] G'abor G'evay, Egon Schulte, J“org M. Wills. The Regular Gr"unbaum Polyhedron of Genus 5. arXiv:1212.6588 [math.CO] 